\title{
Exploring near-infrared absorbing nanocarriers to overcome cancer drug resistance
}

\author{
Siwei Chu, Ursula Stochaj \\ Department of Physiology, Faculty of Medicine, McGill University, Montreal H3G1Y6, Quebec, Canada.
}

Correspondence to: Prof. Ursula Stochaj, Department of Physiology, Faculty of Medicine, McGill University, Montreal H3G1Y6, Quebec, Canada. E-mail: ursula.stochaj@mcgill.ca

How to cite this article: Chu S, Stochaj U. Exploring near-infrared absorbing nanocarriers to overcome cancer drug resistance. Cancer Drug Resist2020;3:302-33. http://dx.doi.org/10.20517/cdr.2020.20

Received: 5 Apr 2020 First Decision: 21 Apr 2020 Revised: 2 May 2020 Accepted: 18 May 2020 Available online: 2 Jul 2020

Science Editor: Vladimir P. Torchilin Copy Editor: Cai-Hong Wang Production Editor: Tian Zhang

\begin{abstract}
One of the major obstacles of successful cancer therapy is cancer drug resistance. The unique tools and applications developed by nanomedicine provide new approaches to surmount this common limitation of current treatment regimens. Nanocarriers that absorb light in the near-infrared spectrum are particularly suitable for this purpose. These nanocarriers can produce heat, release drugs or stimulate the production of physiologically relevant compounds when illuminated with near-infrared light. The current review summarizes the causes contributing to cancer multidrug resistance. The major types of nanocarriers that have been developed in recent years to overcome these hurdles are described. We focus on nanoparticles that are responsive to near-infrared light and suitable to surmount cancer multidrug resistance. Our review concludes with the bottlenecks that currently restrict the use of nanocarriers in the clinic and an outlook on future directions.
\end{abstract}

Keywords: Cancer drug resistance, chemotherapy, nanomedicine, near-infrared light, combination therapy, photothermal therapy, photodynamic therapy, controlled drug release

\section{INTRODUCTION}

Drug resistance, a persistent bottleneck for cancer therapy

At present, surgery, radiation and chemotherapy are the leading regimens for cancer treatment which are often combined with targeted or immunotherapy ${ }^{[1]}$. The advantages of chemotherapy include highly standardized treatment protocols, and extensive experience has been gained with their clinical applications. However, drug-resistant cancer cells can be inherent to the cancer or are acquired during therapy. Multidrug resistance (MDR) in particular compromises treatment efficacy and promotes disease recurrence and cancer-related patient death ${ }^{[2]}$. MDR has been defined as the "resistance of cancer cells

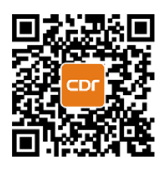


to structurally and mechanistically unrelated classes of anticancer drugs ${ }^{9[3]}$. Treatment resistance is not limited to chemotherapy, but also applies to targeted and immunotherapy ${ }^{[4]}$. Dose escalation has been used to counter drug resistance, but it fails to eliminate all tumor cells ${ }^{[5]}$. Moreover, the increased concentration of cancer drugs is often associated with severe side effects. While elevated dose intensity regimens limit the recurrence of some cancers ${ }^{[5]}$, alternative or complementary strategies are needed to combat cancer drug resistance. Nanocarriers are especially promising tools for the development of such complementary approaches. To maximize the efficacy of nanocarriers, it is important to consider the different origins of cancer drug resistance.

\section{Mechanisms of cancer drug resistance}

The causes of treatment resistance are not limited to the tumor cells. They are often multifactorial ${ }^{[2]}$ and include the complex tumor microenvironment (TME). The heterogeneity of cancer cells within the tumor, temporal changes in tumor cell properties, and differences among metastases complicate the design of proper treatment regimens.

Antineoplastic resistance may be intrinsic to the tumor or develop during therapy. A network of factors collaborates to promote treatment resistance; such resistance can be obtained through pathway reactivation, bypass or indifference ${ }^{[2]}$. Cancer cells acquire drug resistance through mutations in key signaling molecules ${ }^{[6]}$ or non-genetic changes ${ }^{[7]}$. Chemoresistance is linked to diverse targets that control drug transport, signaling and metabolic routes; they determine cancer cell survival and proliferation ${ }^{[2,8]}$. Notably, a subset of these targets is non-druggable (see Future Directions). Non-druggable and particularly important to therapy are mutations in genes encoding oncogenic drivers, as exemplified by MYC, RAS, TP53, and $B R A F^{[9]}$. In addition, cancer stem cells (CSCs), a subpopulation of tumor cells with self-renewal capabilities, often display greatly elevated treatment resistance ${ }^{[10,11]}$.

Cancer cell and CSC survival is promoted by reduced intracellular drug concentrations, suppressed apoptosis, enhanced damage repair, and changes in the abundance of drug targets ${ }^{[11-13]}$. Changes in drug transport and metabolism represent major paths to chemotherapeutic failure ${ }^{[12]}$. Specific mechanisms contributing to MDR include the activation of anti-apoptotic signaling, inactivation of pro-apoptotic signals, environment-mediated drug resistance ${ }^{[14]}$, cell dormancy ${ }^{[15]}$, altered microRNA profiles, and the production of extracellular vesicles ${ }^{[16]}$. In the context of near-infrared (NIR) absorbing nanocarriers, research has focused mainly on intracellular drug concentrations, which are determined by drug uptake, metabolism and efflux.

\section{$A B C$ transporters}

Cancer drug efflux mediated by the ATP-binding cassette (ABC) transporter family is especially well understood $^{[3,17,18]}$. ABC family members move structurally diverse compounds across cellular membranes. Among the $\mathrm{ABC}$ transporter substrates are agents that impinge on various cancer survival pathways. Besides common chemotherapeutic agents, several ABC transporters also mediate the efflux of compounds used for molecularly targeted therapy ${ }^{[19]}$.

The overexpression of several $\mathrm{ABC}$ transporters stimulates cancer drug efflux through an ATP-dependent process $^{[3]}$. Notably, overabundant ABC transporters promote MDR in tumor cells; their overexpression can be particularly high in CSCs ${ }^{[15]}$. Various ABC transporters are critical contributors to MDR, especially members of the ABCB, ABCC, and ABCG subfamily ${ }^{[3,17,20]}$. Several key players have been identified: (1) permeability-associated glycoprotein (P-gp, P-glycoprotein, ABCB1, encoded by the MDR1 gene); (2) multidrug resistance-associated protein 1 (MRP1, ABCC1); (3) breast cancer resistance protein (BCRP, ABCG2); and (4) MRP2 (ABCC2). 


\section{P-Glycoprotein}

To date, P-gp is one of the most common host factors for cancer MDR that has been targeted with nanoparticles (NPs). Like other ABC transporters, P-gp is an integral membrane protein that is critical for the transport of diverse cargos across cell membranes ${ }^{[21]}$. P-gp is particularly abundant in the plasma membrane of cells that excrete physiological substrates and xenobiotics ${ }^{[22]}$. P-gp resides in epithelial cells of the intestine, renal proximal tubules, liver bile ductules, and other cell types ${ }^{[3,17,22]}$. P-gp cargos include a wide spectrum of endogenous substrates, such as hormones, cytokines, chemokines, and nutrients ${ }^{[23]}$. Moreover, P-gp transports neutral and cationic hydrophobic compounds that are used in the clinic. Specific examples are taxanes (such as docetaxel), anthracyclines (doxorubicin), antibiotics, and tyrosine kinase inhibitors ${ }^{[3,17,24]}$. Thus, P-gp affects the pharmacokinetics of numerous drugs ${ }^{[3,17,24]}$.

P-gp activities have multiple consequences for therapy. P-gp (1) restricts the intestinal absorption of drugs, (2) controls drug passage through the blood-brain-barrier, and (3) promotes drug excretion through renal and hepatobiliary routes ${ }^{[2]}$. Hence, P-gp activities can profoundly diminish the cytotoxicity of therapeutic agents. Taken together, P-gp regulates the systemic concentration of many chemotherapeutic compounds and thereby often contributes to MDR.

The organization and molecular functions of P-gp have been studied extensively ${ }^{[24,26]}$. In brief, alternative splicing and single nucleotide polymorphisms produce several P-gp isoforms. Human P-gp consists of 1276-1280 amino acid residues which are organized into two repeating units. Each individual unit contains a cytoplasmic nucleotide-binding domain (binds ATP) and a transmembrane domain. The two units collaborate in the P-gp transport cycle. While the transmembrane domains bind the substrate, the nucleotide-binding domains are required for ATP binding and hydrolysis ${ }^{[26]}$.

Several strategies have been explored to control P-gp activity. This can be achieved by modulating P-gp (1) substrate binding and transport, (2) ATPase cycle, (3) membrane environment, (4) protein stability, (5) gene expression, and (6) mRNA translation. To date, different generations of $\mathrm{ABC}$ transporter inhibitors have been synthesized and evaluated. However, they have limited efficiency ${ }^{[17]}$ and often display off-target effects $^{[27]}$. As these compounds are unlikely to overcome MDR, alternative strategies are being developed. Several of these strategies rely on nanocarriers that respond to NIR light.

\section{Other molecular factors promoting cancer multidrug resistance}

Aside from $\mathrm{ABC}$ transporters, additional cellular factors facilitate MDR through mechanisms that are driven by protein-protein interactions or gene regulatory networks ${ }^{[28-30]}$. For example, transcriptional regulators and epigenetic mechanisms determine de novo gene expression, which can ultimately enhance MDR. As such, the transcription factor NF- $\mathrm{BB}$ and mutant 53 control MDR1 gene expression and thereby modulate the response to chemotherapeutic drugs. Notably, both NF- $\mathrm{KB}$ and mutant $\mathrm{p} 53$ are targetable with NIR-responsive $\mathrm{NPs}^{[31]}$ (see section on gold nanoparticles). Other factors linked to MDR include target mutations, drug metabolism, increase in repair enzymes, antioxidant components, and molecular chaperones ${ }^{[30,32]}$. Regulated in part by the TME, pH, oxygen, and nutrient supply of the cancer cells also shape the response to chemotherapeutic agents ${ }^{[16]}$. For example, hypoxia-inducible transcription factors (HIF-1, HIF-2) can upregulate the expression of genes encoding drug efflux pumps ${ }^{[16]}$. At present, several components relevant to MDR have yet to be incorporated in NP-based strategies (see Future Directions).

Resistance to doxorubicin, cisplatin or docetaxel

Many of the studies related to cancer MDR and nanomedicine concentrate on the resistance to doxorubicin ${ }^{[33-35]}$, cisplatin ${ }^{[34,36,37]}$, or docetaxel ${ }^{[38-42]}$. These drugs are frequently used for research on stimulus-responsive NPs. In the clinic, the anthracycline doxorubicin [Figure 1] is applied to treat a variety of malignancies ${ }^{[35]}$. Several biological processes are associated with the resistance to anthracyclines, 


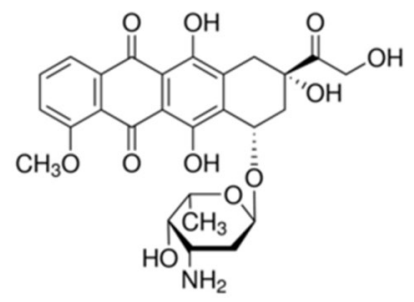

Doxorubicin

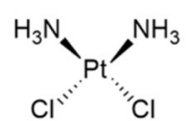

Cisplatin

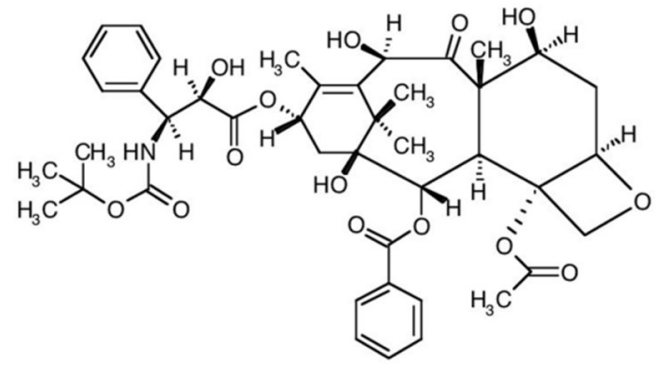

Docetaxel

Figure 1. Chemical structures of doxorubicin, cisplatin, and docetaxel

including drug transport, topoisomerase II activity, DNA repair, cancer stemness, and drug metabolism ${ }^{[35]}$. In the context of stimulus-responsive NPs, doxorubicin-related studies have focused predominantly on drug efflux. Several ABC transporters are implicated in doxorubicin efflux, such as P-gp (ABCB1), MRP1 $(\mathrm{ABCC} 1)$ and BCRP $(\mathrm{ABCG} 2)^{[3,35]}$. Depending on the type of cancer, additional carriers may add to doxorubicin resistance ${ }^{[35]}$.

A large number of cancers are treated with cisplatin. Cisplatin resistance is determined by multiple factors that fall into three major categories: DNA repair, intracellular accumulation, and drug modifications in the cytoplasm ${ }^{[37]}$. Various membrane transporters participate in the uptake and efflux of cisplatin and additional platinum compounds ${ }^{[37,43-45]}$. The copper transporters CRT1 and CTR2, as well as members of the organic cation transporter family (OCT) facilitate cisplatin uptake ${ }^{[37,43]}$. MRP2 and other transmembrane proteins have been linked to cisplatin efflux ${ }^{[34,37]}$.

Microtubule stabilizers and destabilizers are widely used for the treatment of malignant solid tumors ${ }^{[38]}$. Docetaxel is a taxane that binds $\beta$-tubulin, which stabilizes microtubules and prevents the completion of cell division ${ }^{[38-42]}$. Several mechanisms contribute to docetaxel resistance. These include mostly efflux through P-gp and other ABC transporters, mutation of the drug-binding site of $\beta$-tubulin, and the synthesis of an alternative $\beta$-tubulin isoform ${ }^{[3,38,42]}$. Further routes to docetaxel resistance have been identified $^{[42]}$.

\section{Strategies to overcome drug resistance in the clinic}

The complexity and heterogeneity of tumors, even within the same patient, pose major obstacles for the selection of appropriate treatment regimens. Precision medicine applies the genetic information on the patient's cancer and pharmacogenomics to optimize targeted treatment ${ }^{[16,46-49]}$. This bio-guided approach relies on genetic information on molecular markers to block oncogenic pathways ${ }^{[48]}$. Specifically, identifying genetic variants of oncogenes or tumor suppressors aids in selection of the most appropriate chemotherapy for each patient. This is crucial as the response to drug treatment is highly variable and to a large extent driven by genetic variants ${ }^{[16]}$. Pharmacogenomics Knowledgebase $\left(\right.$ PharmGKB $^{[50]}$ ) provides information on drug-gene interactions and thus helps to identify the most suitable approach for targeted therapy.

\section{Nanomedicine for cancer therapy}

The unique properties of nanomaterials have encouraged the development of nano-based theranostics. Drug delivery through nano-sized carriers can lead to marked changes in whole-body distribution, pharmacokinetics, and pharmacodynamics of the agent. Patients may further benefit from the intrinsic therapeutic properties of some nanomaterials ${ }^{[51]}$. Numerous nanomaterials are currently evaluated for health applications ${ }^{[52]}$. In particular, NPs with defined composition, size, morphology (e.g., spheres, hexagons, rods, sheets), and surface coating are being assessed for diagnosis and therapy. As discussed in 
the following section, NP-based nanomedicine provides distinctive advantages that are not always available through other applications.

\section{Extending drug circulation time and bypassing drug efflux}

A major rationale for the development of therapeutic NPs is that NP-mediated drug delivery can reduce the treatment-associated toxicity. Indeed, NP-assisted drug delivery offers several therapeutic benefits, especially in the context of cancer drug resistance. First, free anti-cancer drugs often have a short systemic circulation time. Before reaching the desired sites, they may be removed from the circulation, mainly through interstitial diffusion, non-specific binding, glomerular filtration, as well as hepatic clearance ${ }^{[53]}$. At the cellular level, drug metabolism and efflux are major contributors to drug clearance. However, when protected by NP association, chemotherapeutic drugs can maintain a higher concentration and prolonged residence in the circulatory system ${ }^{[54]}$.

Second, drug efflux opposes the accumulation of free drugs in cancer cells. It is typically mediated by transporters located in the plasma membrane (see above). By contrast, cells often internalize drug-NP complexes through endocytic pathways. These routes differ fundamentally from transporter-mediated uptake, as they include clathrin- or caveolae-mediated endocytosis and rely on vesicle trafficking ${ }^{[55]}$.

Enclosed in endosomal vesicles, NP-associated drugs are protected against efflux from the cell. Moreover, during vesicle trafficking, drug release can be accomplished at subcellular locations where efflux pumps are sparse. Together, these mechanisms increase intracellular drug concentrations. Importantly, NP-based drug formulations often reduce side effects, which improves patient adherence to therapy and thus overall treatment success. Targeting NPs to desired locations (see next section) can further improve NP-based therapy.

\section{Targeted drug delivery}

NPs can target tumors and the TME through passive or active processes ${ }^{[56]}$. Various approaches have been explored to enhance NP accumulation at these sites. However, it should be emphasized that improved NP delivery to the tumor or TME is only meaningful in the clinic if it increases treatment success ${ }^{[57,58]}$. Enhanced permeability and retention (EPR) has been described as the main basis for passive targeting to solid tumors, but the importance of EPR for the treatment of human tumors continues to be debated ${ }^{[57,58]}$. A recent study points to alternative mechanisms and highlights the role of transcytosis across endothelial cells for NP extravasation into the tumor ${ }^{[59]}$. Active tumor targeting has been accomplished by various NP modifications. Conjugation to antibodies, ligands, or aptamers has directed NPs to the surface of malignant cells or to the $\mathrm{TME}^{[56,60,61]}$.

Both passive and active tumor targeting strategies can enhance drug supply to the tumor site, which affords important therapeutic benefits. First, drug concentrations increase in cancer cells. Second, with elevated concentrations in the tumor, systemic drug doses can be diminished. This in turn may reduce side effects. A recent phase I trial in human patients with solid tumors demonstrated the benefits of NP-mediated drug delivery ${ }^{[62]}$. Compared with the free drug (docetaxel), docetaxel-loaded NPs increased intratumoral drug concentrations, while significantly diminishing adverse outcomes ${ }^{[63]}$.

\section{Stimulus-responsive nanoparticles for cancer therapy}

Nanomaterials that respond to specific stimuli are interesting for therapy as their functionalities can be controlled by specific cues. As a result, stimulus-responsive NPs offer excellent spatiotemporal control of their functionalities. Such control can be achieved through internal or external cues [Table 1] ${ }^{[58,64]}$. For example, $\mathrm{pH}$, redox state, and enzymatic activities of subcellular organelles, tissues or organs can provide internal triggers for NP-dependent actions. These parameters are relevant to cancer therapy as tumor 
Table 1. Control of nanoparticle functionalities

\begin{tabular}{|c|c|c|}
\hline Stimulus & Internal (provided by cells, tissues, extracellular milieu) & External \\
\hline Light & NA & $\begin{array}{l}\text { Natural light exposure of skin, retina; } \\
\text { targeted light exposure via laser or other artificial } \\
\text { light sources; different wavelengths (UV, visible, } \\
\text { NIR); photo-acoustic therapy }\end{array}$ \\
\hline $\mathrm{pH}$ & $\begin{array}{l}\text { Tumor microenvironment }{ }^{[65]} \text {; } \\
\text { tissues, organs, biological fluids: } \\
\text { stomach ( } \mathrm{pH} 1.5-3.5 \text { ), intestine (slightly alkaline), blood ( } \mathrm{pH} ~ 7.3-7.5) \text {, } \\
\text { lymph (slightly alkaline); } \\
\text { subcellular organelles with low pH: lysosomes, endosomes, Golgi } \\
\text { apparatus (pH 4.0-6.5) }\end{array}$ & NA \\
\hline Redox environment & $\begin{array}{l}\text { Endoplasmic reticulum (oxidative protein folding } \\
\text { TME may increase oxidative stress in tumor cells } s^{[65]}\end{array}$ & NA \\
\hline Heat & Body temperature & $\begin{array}{l}\text { Extrinsic trigger to change temperature of biological } \\
\text { environment; } \\
\text { laser irradiation at different wavelengths }{ }^{[69]} \text {; } \\
\text { alternating magnetic fields (induction) }{ }^{770]}\end{array}$ \\
\hline Cold & NA & Cold-induced drug release $\mathrm{e}^{[77]} ;$ cryosurgery $^{[72]}$ \\
\hline Ultrasound & NA & Disintegration of NP clusters ${ }^{[73]}$ \\
\hline Metabolites & $\begin{array}{l}\text { Glutathione, increased concentrations in tumor cells; } \\
\text { lactate, elevated concentrations in tumor and TME }\end{array}$ & NA \\
\hline Magnet & NA & External magnetic field \\
\hline
\end{tabular}

Stimuli that have been used to regulate nanoparticle functionalities are depicted. The list is not comprehensive; it focuses on triggers that are well-established for nanomedical approaches. NIR: near-infrared; TME: tumor microenvironment

cells and the TME are generally more acidic. They also exhibit altered redox properties and enzymatic activities $^{[64-66]}$.

The extrinsic control of NP performance differs in several aspects from intrinsic regulation. Extrinsic triggers can limit off-target effects in healthy tissues and have the potential for precise eradication of malignant cells $^{[58]}$. However, this requires that tumor size and location are known, which is difficult if tumors or metastases are small or spread over multiple anatomical locations. Thus, NPs controlled by external stimuli will be particularly useful to treat localized tumors that are not resectable.

External triggers, especially light, have been employed to regulate diverse NP functionalities. Relevant to cancer treatment are NP-based drug sequestration, stabilization, and delivery. In addition, the NPmediated heat or ROS production and enzyme activation have also been explored [Table 1].

\section{Light-responsive nanoparticles}

Wavelengths throughout the electromagnetic spectrum have been employed to control stimulus-dependent $\mathrm{NPs}^{[74,75]}$. However, there are restrictions for in vivo applications, because UV and visible light is strongly absorbed by biological material ${ }^{[7,76]}$. Possible consequences are damage to biomolecules, elevated tissue temperature, and light scattering accompanied by reduced tissue penetration ${ }^{[77-79]}$. By contrast, NIR irradiation can achieve deeper tissue penetration ${ }^{[75,79-81]}$. The NIR window between $700 \mathrm{~nm}$ and 1,000 $\mathrm{nm}$ is ideal for light-mediated therapeutics. Depending on the power density and properties of the biological material, NIR may penetrate to a depth of $3 \mathrm{~cm}^{[75,82]}$. This value may be limited to unique conditions, as many publications suggest a penetration depth of $1 \mathrm{~cm}$ or less ${ }^{[79,81]}$. Crucial for therapy, NIR exposure is associated with low toxicity for healthy tissues ${ }^{[83]}$. Although NIR laser light can be phototoxic at high doses, genotoxic and mutagenic effects are likely minor ${ }^{[84]}$. Nevertheless, at high laser irradiance, NIR may increase the local temperature of biological specimens and produce reactive oxygen species $(\mathrm{ROS})^{[84]}$. On the other hand, this heat and ROS production can be exploited for tumor cell eradication. Indeed, both modalities have been incorporated in nanotherapeutic regimens (see below). 


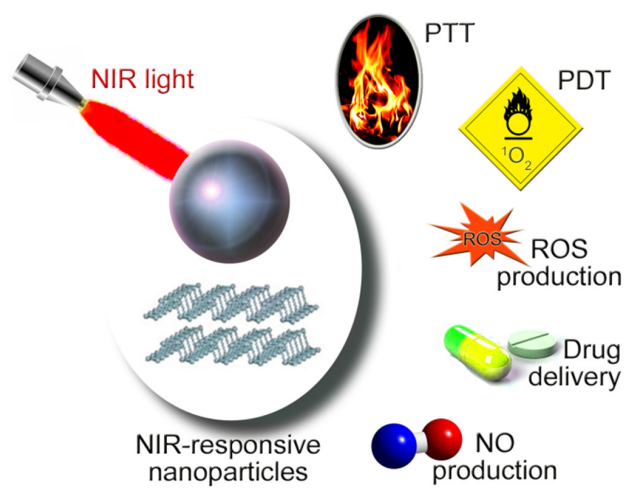

Figure 2. NIR-responsive NP actions relevant to cancer MDR. NIR-responsive NPs have been evaluated for their ability to surmount the drug resistance of cancer cells. Spherical NPs and nanosheets are depicted as examples. A set of NP-based treatment modalities has been explored in vitro and in vivo. PTT, PDT, ROS production, drug delivery, and NO production are the main applications that have been examined to date. NIR: near-infrared; NP: nanoparticle; MDR: Multidrug resistance; PTT: photothermal therapy; PDT: photodynamic therapy; ROS: reactive oxygen species; NO: nitric oxide

\section{Therapeutic applications of stimulus-responsive nanoparticles}

This section of the review focuses on NIR as an external trigger to activate NP-based activities. Figure 2 illustrates the major functionalities of NIR-responsive NPs that have been assessed for the elimination of MDR cancer cells.

\section{Photothermal therapy}

Photothermal therapy (PTT) is a minimally invasive treatment modality that locally elevates cell or tissue temperature. Several molecular mechanisms make cancer cells more sensitive to heat than their normal counterparts ${ }^{[85,86]}$. These differences are clinically relevant and have been exploited for PTT. Specifically, localized heat generation destroys cancer cells, which may include drug-resistant tumor cells. The most common modes of PTT-induced death are apoptosis and necrosis. While photothermal ablation of the tumor is often incomplete ${ }^{[87]}$, heat is nevertheless beneficial to combat MDR. It may re-sensitize resistant cancer cells to chemotherapy through different pathways. These routes include up-regulating the abundance of heat shock factor (HSF-1) or decreasing P-gp levels ${ }^{[88]}$.

Depending on the nanomaterial properties, the intensity and duration of irradiation, NIR light can increase NP temperatures to $40-50{ }^{\circ} \mathrm{C}$ and above ${ }^{[87,89,90]}$. NIR-absorbing organic small molecules can also serve as sensitizers for PTT. When incorporated in nanocarriers they can provide or enhance PTT modalities ${ }^{[91]}$. The nanomaterials discussed here differ widely in their ability to generate and withstand heat. For example, platinum-containing NPs have extreme heat stability ${ }^{[92]}$. However, it is not clear whether this is relevant to clinical applications, especially since temperatures $>100{ }^{\circ} \mathrm{C}$ reduce the efficiency of thermal tumor ablation ${ }^{[8]}$.

\section{Photodynamic therapy}

Photodynamic therapy (PDT) exploits the cytotoxicity of reactive molecular species (ROS; reactive nitrogen species, RNS). The treatment requires the administration of a photosensitizer, which absorbs light at a defined wavelength, a light source, and oxygen (or another substrate) ${ }^{[93-95]}$. Following irradiation, elevated levels of reactive molecular species have twofold impact on the tumor; they may kill cancer cells directly, or affect the TME, especially tumor vessels. The major routes of cancer cell death caused by PDT include apoptosis, necrosis, autophagy-dependent death, and paraptosis ${ }^{[94,95]}$. Interestingly, the PDTinduced ROS production may damage some of the $\mathrm{ABC}$ transporters, which can diminish $\mathrm{MDR}^{[96]}$. Like thermal ablation ${ }^{[85]}$, PDT has been associated with enhanced adaptive immunity or immunosuppression ${ }^{[94]}$. 
A key step for PDT is the efficient delivery of photosensitizers to the tumor sites. Photosensitizers are often hydrophobic and prone to aggregation, but their delivery by nanocarriers can circumvent this difficulty ${ }^{[95]}$.

\section{Controlled drug release}

NIR light can trigger drug release from nanocarriers in a confined space and highly regulated temporal fashion. The strategy of controlled drug release $(\mathrm{CDR})^{[97]}$ elevates drug concentrations at tumor sites, without a concomitant overall increase in the circulatory system. The localized rise in drug concentration at the tumor sites is advantageous in the context of MDR, as it could exceed the capacity of efflux pumps. A potential outcome is the killing of chemoresistant cancer cells. Moreover, the drug-related side effects will be limited, as healthy tissues and organs have only limited exposure to chemotherapeutic agents.

\section{Nitric oxide generation}

Doxorubicin, a commonly used chemotherapy drug, stimulates nitric oxide (NO) production, in part through the increase in NO synthase activity ${ }^{[98]}$. NO synthesis contributes to the cytotoxicity of doxorubicin $^{[98]}$, but is diminished in doxorubicin-resistant cells ${ }^{[99]}$. Notably, increased NO concentrations impair drug efflux pumps ${ }^{[99]}$, which reduces MDR. The combination of NO donors with NIR-responsive nanocarriers exploits this effect and facilitates drug accumulation in MDR cancer cells.

\section{Combination therapy}

In combination therapy, two or more treatment modalities are used together to improve treatment success. These modalities may target different cellular mechanisms that contribute to cancer cell survival and proliferation ${ }^{[100]}$. By hitting multiple targets, the simultaneous modulation of several signaling pathways or synthetic-lethal strategies increase the likelihood of cancer cell killing ${ }^{[100]}$.

NP-based combination therapy offers advantages over other approaches. For example, when multiple drugs are combined, differences in the pharmacokinetics may prevent optimal treatment outcome for drug combinations ${ }^{[101]}$. Nanomedicine may surmount this hurdle, as NP-based co-encapsulation supports synchronized drug delivery to the tumor. Furthermore, PDT-induced killing of cancer cells can be enhanced when photosensitizers are loaded onto NPs and released in a localized manner ${ }^{[94]}$. NP-based combination therapy is also promising to combat cancer drug resistance. To achieve this, NPs have served as carriers for efflux pump inhibitors ${ }^{[29,102,103]}$, siRNAs, pro-apoptotic molecules, therapeutic antibodies, and other agents ${ }^{[104,105]}$. The combination of thermal ablation with other therapeutic modalities is especially promising for NP-dependent approaches ${ }^{[106]}$.

\section{NEAR-INFRARED LIGHT ABSORBING NANOMATERIALS FOR CANCER THERAPY}

A variety of nanomaterials with strong NIR absorption have been developed and tested in recent years. Depending on the selection of NPs and settings for NIR irradiation, different mechanisms contribute to light-induced drug release. On the level of NPs they include NIR-triggered disintegration, destruction of drug-carrier bonds, and removal of 'gate-keepers' from mesoporous $\mathrm{NPs}^{[107]}$. The use of pulsed vs. continuous wave NIR also determines the impact on cancer cell viability, which has been attributed in part to differences in drug release ${ }^{[108]}$. By combining the advantages of NP-assisted drug delivery and lightcontrolled drug release with PTT or PDT, NIR-absorbing NPs have great potential to defeat cancer drug resistance. In the following section, we briefly describe in vitro and in vivo models that are commonly used for nanomedical research in the context of drug resistance [Table 2]. NIR-absorbing nanocarriers are then introduced as tools for cancer therapy and specific examples discuss NP-based approaches to overcome MDR.

\section{Cellular and animal model systems to study cancer MDR}

Different cellular and animal models have been developed to investigate tumor cell MDR. Model systems to examine transporter-dependent drug efflux have been reviewed recently ${ }^{[156]}$. Many studies on NIR- 
Table 2. Frequently used cell lines and compounds to evaluate NIR-responsive nanoparticles in the context of multidrug resistance

\begin{tabular}{|c|c|}
\hline $\begin{array}{l}\text { Cell line, targeting moieties, drugs, } \\
\text { other reagents }\end{array}$ & Properties, references \\
\hline $\begin{array}{l}\text { "MCF-7/AD", re-classified as NCI/ADR-RES; } \\
\text { 2D cultures and 3D spheroids; also used in } \\
\text { different mouse models }\end{array}$ & Doxorubicin-resistant; derived from human ovarian carcinoma (OVCAR-8) cells ${ }^{[109,110]} ;$ MDR $^{[88,111-129]}$ \\
\hline SW620/Ad300 cells & Doxorubicin-resistant; obtained from human colorectal adenocarcinoma cells SW620; MDR ${ }^{[102,130]}$ \\
\hline HT29-dox cells & Doxorubicin-resistant; obtained from human colon epithelial cells HT29; MDR ${ }^{[99]}$ \\
\hline A2780cisR cells & Human ovarian carcinoma; cisplatin-resistant; $\operatorname{MDR}^{[131,132]}$ \\
\hline SCC-7 cells & Mouse, squamous cell carcinoma cells ${ }^{[111]}$ \\
\hline cos7 cells & African green monkey, kidney fibroblasts ${ }^{[11]]}$ \\
\hline $4 \mathrm{~T} 1$ cells & Mouse, mammary gland carcinoma cells ${ }^{[114]}$ \\
\hline HeLa cells & Human, cervix adenocarcinoma ${ }^{[114]}$ \\
\hline MDA-MB-231 cells & $\begin{array}{l}\text { Human, mammary adenocarcinoma; chemoresistant; overexpression of several ATP } \\
\text { transporters }{ }^{[133,134]}\end{array}$ \\
\hline MDA-MB-453 cells & $\begin{array}{l}\text { Human mammary gland, metastatic carcinoma; high DNA methyltransferase (DNMT) activity; } \\
\text { chemoresistant; hypermethylator phenotype; mutant phosphatase PTEN gene }{ }^{[135-137]}\end{array}$ \\
\hline HepG2cisR cells & Human, hepatocellular carcinoma; cisplatin-resistant, MDR ${ }^{[132]}$ \\
\hline K562R cells & Human, chronic myelogenous leukemia; elevated abundance of P-gp; MDR ${ }^{[138,139]}$ \\
\hline Hyaluronic acid (HA); used for cell targeting & $\begin{array}{l}\text { Natural glycosaminoglycan polysaccharide; binds to CD44 receptor on cell surface; biodegradable } \\
\text { by hyaluronidases }{ }^{[140-142]}\end{array}$ \\
\hline Folate; used for cell targeting & Vitamin B9, also called folic acid; binds to folate receptor on cell surface ${ }^{[143,144]}$ \\
\hline Transferrin & $\begin{array}{l}\text { Iron-binding protein recognized by transferrin receptor (TfR); TfR located on cell surface; TfR } \\
\text { abundance often highly increased in cancer cells }\end{array}$ \\
\hline Doxorubicin & $\begin{array}{l}\text { Multiple modes of action, such as formation of DNA adducts, inhibition of topoisomerase II, DNA } \\
\text { and RNA polymerases; also called adriamycin }{ }^{[33,35]}\end{array}$ \\
\hline Quercetin & $\begin{array}{l}\text { Natural polyphenolic flavonoid present in fruits and vegetables; may have chemopreventive } \\
\text { activity }^{[146]}\end{array}$ \\
\hline 3-Methyl adenine & Inhibits autophagy ${ }^{[147]}$ \\
\hline Chloroquine & Inhibits autophagy ${ }^{[148]}$ \\
\hline D- $\alpha$-Tocopherol polyethylene glycol succinate & P-gp inhibitor ${ }^{[149]}$, also called tocophersolan, TPGS \\
\hline Tetradecanol & Long-chain fatty alcohol ${ }^{[150]}$, undergoes phase change \\
\hline Cisplatin & Produces DNA lesions ${ }^{[36,37]}$ \\
\hline Irinotecan & $\begin{array}{l}\text { Also called camptothecin-11 or CPT-11; activated by carboxylase-converting enzyme, which } \\
\text { generates SN-38; SN-38 inhibits topoisomerase } \mathrm{I}^{[151,152]}\end{array}$ \\
\hline Docetaxel & Binds and stabilizes microtubules ${ }^{[39-41]}$ \\
\hline Poloxamer 188 & Ethylene oxide/propylene oxide copolymer; likely inhibits P-gp ${ }^{[133,153,154]}$ \\
\hline Polyethylene glycol (PEG) & Polymer; used for NP coating ${ }^{[155]}$ \\
\hline
\end{tabular}

Multidrug resistance (MDR) has been demonstrated where indicated. NP: nanoparticle; TPGS: D- $\alpha$-tocopherol polyethylene glycol succinate; NIR: near-infrared

responsive NPs focus on efflux pumps. Several reagents and models are commonly employed by different research groups. They are briefly described here and listed in Table 2.

\section{Cell lines and spheroids}

A large number of studies relied on "MCF-7/ADR" cells. Initially considered a derivative of the human epithelial breast cancer cell line MCF-7, the cells have been re-classified as NCI/ADR-RES ${ }^{[109]}$. It was demonstrated that this cell line originates from human ovarian carcinoma (OVCAR-8) cells. Unlike MCF7 cells, NCI/ADR-RES cells are caspase-3 positive and carry a rare p53 mutation. However, NCI/ADRRES cells do exhibit doxorubicin-resistance, which is crucial to the studies discussed here. For simplicity, we adhere to the nomenclature used in the original publications, but refer to the cells as "MCF-7/ADR" to indicate their re-classification. Other common MDR model systems are SW620/Ad300 and HT29dox cells. The human colorectal adenocarcinoma cell line SW620 was used to generate the drug resistant sub-line SW620/Ad300 ${ }^{[130]}$. HT29-dox is a doxorubicin-resistant derivative of HT29 cells, a human colon epithelial cell line ${ }^{[99]}$. Additional cell lines relevant here are listed in Table 2. Aside from 2D cultures, NIRresponsive NPs are also assessed with $3 \mathrm{D}$ spheroid models of $\mathrm{MDR}^{[157]}$. As spheroids mimic multiple in vivo properties of solid tumors, they are especially valuable to assess NPs and other anti-cancer agents ${ }^{[158-160]}$. In general, cancer spheroids are more resistant to treatment when compared with $2 \mathrm{D}$ cultures ${ }^{[158-160]}$. Several parameters have been identified that determine NP penetration of tumor spheroids ${ }^{[161]}$. 


\section{Experimental animals}

Some studies on MDR and NIR-responsive NPs have been carried out with experimental animals in vivo. In most cases, the research involved mice and heterotopic xenograft models. Tumor cells were often injected subcutaneously or into the tail vein.

\section{Anticancer drugs and other relevant agents}

Many of the NP-based research described here has been performed with the chemotherapeutic agents doxorubicin and cisplatin, whereas the use of docetaxel was less common [Figure 1]. The anthracycline doxorubicin targets DNA topoisomerase $\mathrm{II}^{[162]}$ and has multiple synonyms, such as adriamycin ${ }^{[3,35]}$. Cisplatin and its derivatives cause DNA damage ${ }^{[36,37]}$; the taxane docetaxel stabilizes microtubules ${ }^{[38-42]}$. Some studies also included inhibitors of autophagy or natural compounds [Table 2].

Recent work incorporated phase change material in NIR-responsive NPs ${ }^{[112]}$ to promote the temperaturedependent release of pharmacological agents. For example, 1-tetradecanol has a melting point around 38$39^{\circ} \mathrm{C}$, which is ideal for the NIR-induced discharge of pharmacological compounds.

\section{SURMOUNTING CANCER MDR WITH NIR-RESPONSIVE NANOPARTICLES}

The impact of various NIR-responsive NPs has been examined in drug-resistant cancer cells. The NPs belong to different groups [Table 3]. They have been evaluated in vitro and in vivo; specific examples are described in the following sections and listed in Table 4.

\section{Localized surface plasmon resonance}

Noble metal nanostructures, such as gold (AuNPs), silver (AgNPs), and platinum NPs (PtNPs), are characterized by a strong absorption of visible and near-infrared light. The surface plasmon resonance (SPR) phenomenon provides noble metal NPs with distinctive characteristics that are ideal for phototherapy ${ }^{[172]}$. In brief, NP illumination results in SPR, defined as "a collective oscillation of the metalfree electrons with respect to the NP lattice that is in resonance with the light field" ${ }^{[172]}$. Changes in the shape or morphology of metallic NPs can alter the localized surface plasmon resonance (LSPR) ${ }^{[173]}$.

The LSPR of metal oxide-based nanomaterials also depends on NP size and shape. The LSPR determines the photothermal effect of conductive metal oxides ${ }^{[174]}$. Importantly, the LSPR can be tuned solely through changes in the composition of metal oxide $\mathrm{NPs}^{[174]}$. These properties are particularly useful when small NPs are required, for example to ensure rapid renal clearance.

\section{Noble metal nanoparticles to treat multidrug resistant cancer Gold nanoparticles}

Anisotropic gold NPs of different sizes, morphologies (nanorods, nanostars, nanocages), and surface coatings have been used as NIR-responsive NPs ${ }^{[175]}$. All of these parameters affect NP performance in biological systems ${ }^{[176,177]}$. Gold nanorods (AuNRs) generate two plasmon bands ${ }^{[177]}$, and their anisotropic shape improves tumor accumulation when compared with gold nanospheres ${ }^{[178]}$. Gold nanostars (AuNSs) are especially useful for theranostics, because the electromagnetic field is markedly enhanced at "spikes" In addition, the sharp tips of AuNSs may cause physical damage to cellular structures and organelles ${ }^{[180]}$. Several properties distinguish gold nanocages (AuNCs) from other gold $\mathrm{NPs}^{[181]}$. Thus, AuNCs provide interior space for drug loading and release. At the same time, their mechanical properties are ideally suited for biological environments.

Several studies demonstrate that the photothermal effect of AuNPs can counteract MDR. AuNRs containing a mesoporous silica shell $\left(\mathrm{Au} @ \mathrm{SiO}_{2}\right)$ loaded with doxorubicin re-sensitized drug-resistant "MCF-7/ADR" cells to drug treatment ${ }^{[88]}$. The hyperthermia generated by NIR irradiation of ${\mathrm{Au} @ \mathrm{SiO}_{2}}$ increased the abundance of trimeric HSF-1. The transcription factor HSF-1 is a well-established regulator 
Table 3. NIR-absorbing nanomaterials with potential for NP-based health applications

\begin{tabular}{lll}
\hline Nanoparticle group & \multicolumn{1}{c}{ Nanoparticle subgroups } & \multicolumn{1}{c}{ Ref. } \\
\hline Noble metals & Gold, silver, platinum, palladium & {$[88,102,111,113,114,163,164]$} \\
Lanthanides & Upconverting NPs & {$[115,123,126,131,165]$} \\
Metal chalcogenides & Copper chalcogenides, transition metal dichalcogenides & {$[117,166-170]$} \\
Metal oxides & Iron oxide, titanium oxide, tungsten oxide & {$[157,171]$} \\
Carbon & Carbon nanotubes, carbon nanospheres, graphene oxides & {$[124,125]$} \\
Polymers & Polydopamine & {$[120,127-129]$} \\
Black phosphorus & NA & {$[120,132]$} \\
\hline
\end{tabular}

Near-infrared (NIR)-responsive nanomaterials listed are pertinent to the treatment of cancer multidrug resistance (MDR) and discussed in this review. References indicate successful applications, using in vitro and/or in vivo MDR models. Some composite nanoparticles (NPs) fall into multiple classes

Table 4. Representative NIR-absorbing nanocarriers used to overcome cancer drug resistance. The references provide a detailed composition of the nanoplatforms

\begin{tabular}{|c|c|c|c|c|c|}
\hline Category & Platform & Application & Drug & NIR (nm) & Ref. \\
\hline \multirow[t]{8}{*}{ Noble metals } & AuNS-pep-HA & PTT & DOX & 808 & {$[111]$} \\
\hline & AuNR-CuS-Liposome & NO generation & DOX & 808 & [238] \\
\hline & PLGA-AuHS-death receptor4 & PTT & DOX & 808 & [31] \\
\hline & TD-AuNC-PEG-Biotin & PDT, CDR & DOX, QUR & 808 & [112] \\
\hline & AuNP-SiO ${ }_{2}$ & PTT & DOX & 780 & {$[88]$} \\
\hline & AuNR-mSiO ${ }_{2}$-PHIS-TPGS & PTT, CDR & DOX & 808 & [102] \\
\hline & PtNP-Fucoidan & PTT & DOX & 808 & [113] \\
\hline & CuPd & PTT & DOX, CQ, 3-MA & 808 & [114] \\
\hline \multirow[t]{3}{*}{ Lanthanides } & $\mathrm{NaYF}_{4}: \mathrm{Yb} / \mathrm{Tm}-\mathrm{TiO}_{2}-\mathrm{FA}$ & PDT & DOX & 980 & [115] \\
\hline & $\mathrm{NaGdF}_{4}: \mathrm{Yb} / \mathrm{Nd}-\mathrm{NaGdF}_{4}: \mathrm{Yb} / \mathrm{Er}-\mathrm{NaGdF}_{4}$ & PDT & $\mathrm{RB}, \mathrm{Pt}(\mathrm{IV})$ prodrug & 808 & [131] \\
\hline & $\mathrm{NaGdF}_{4}: \mathrm{Tm} / \mathrm{Yb}-\mathrm{NaGdF}_{4}-\mathrm{OA}$-Azo-Lipo & CDR & DOX & 980 & [123] \\
\hline \multirow[t]{2}{*}{ Copper chalcogenides } & $\mathrm{CuS}-\mathrm{mSiO}_{2}$-SNO & NO generation & DOX & 808 & [116] \\
\hline & $\mathrm{Cu}_{2-x} \mathrm{Te} N C-P E G$ & PTT, PDT & DOX & 808 & [136] \\
\hline Transition metal dichalcogenides & $\mathrm{MoS}_{2}-\mathrm{PEI}-\mathrm{HA}$ & PTT & DOX & 808 & [117] \\
\hline Metal oxides & $\mathrm{Fe}_{3} \mathrm{O}_{4}-\mathrm{PDA}-\mathrm{mSiO}_{2}$ & NO generation & DOX & 808 & [118] \\
\hline \multirow[t]{5}{*}{ Carbon-based } & Graphene oxide-PEG-DA & PTT & DOX & 808 & [119] \\
\hline & Graphene oxide & PTT & DOX, irinotecan & 808 & [133] \\
\hline & Single-walled carbon nanotubes-Ap & PTT & DOX & ns & [138] \\
\hline & Single-walled carbon nanotubes & PTT & DOX & 808 & [125] \\
\hline & Hollow carbon nanospheres & PTT, CDR & DOX & 780 & [124] \\
\hline \multirow[t]{3}{*}{ Polydopamine } & PLGA-PDA-TPGS & PTT, CDR & DTX & 808 & [129] \\
\hline & PDA-PEG-FA & PTT, CDR & DOX, BNN6 & 808 & [128] \\
\hline & PNOC-PDA & PTT, CDR & DOX & 808 & [127] \\
\hline \multirow[t]{3}{*}{ Black phosphorus } & BP-PDA-PEG-Apt & PTT, CDR & DOX & 808 & [120] \\
\hline & Pt-BP & PTT & Cisplatin & 808 & [132] \\
\hline & BP-PDA-PEG-PEITC & PTT, PDT & DOX & 808 & [121] \\
\hline
\end{tabular}

Near-infrared (NIR) refers to the wavelength that was used to trigger a nanoparticle (NP) response. NO: nitric oxide; CDR: controlled drug release; CQ: chloroquine; DA: 2,3-dimethylmaleic anhydride; DOX: doxorubicin; 3-MA: 3-methyl adenine; ns: not specified; PDT: photodynamic therapy; PTT: photothermal therapy; QUR: quercetin; RB: rose bengal

of stress responses. Trimeric HSF-1 accumulated in nuclei, which ultimately reduced the abundance of NF$\kappa \mathrm{B}^{[182]}$. The combination of $\mathrm{Au} @ \mathrm{SiO}_{2}$ with NIR irradiation also diminished the levels of P-gp and mutant p5 $3^{[88]}$. Taken together, NIR illumination of $\mathrm{Au} @ \mathrm{SiO}_{2}$ initiated multiple processes that led to elevated drug sensitivity and reduced viability of "MCF-7/ADR" cells ${ }^{[88]}$.

Independent studies confirmed the efficacy of AuNR-based applications in colorectal cancer cells ${ }^{[102]}$. This was accomplished with an AuNR nanocomposite containing poly-histidine and a mesoporous silica coat for doxorubicin loading. Poly-histidine binds the P-gp inhibitor D- $\alpha$-tocopherol polyethylene glycol succinate (TPGS) in a pH-dependent fashion and enhances NP release from endo-/lysosomes. The 
composite NP was successful for combined chemo- and photothermal therapy (chemo-PTT) of drugresistant colorectal cancer cells (SW620/Ad300) in vitro and in experimental mice ${ }^{[102]}$.

AuNSs have been used to produce subcellular PTT. These NPs functioned as a localized "nanoheater" and drug delivery vehicle ${ }^{[111]}$. The approach involved multiple AuNS modifications to ensure the proper NP distribution and drug delivery: (1) hyaluronic acid, (2) a mitochondrial targeting peptide, and (3) doxorubicin. Hyaluronic acid (HA) functions as active targeting ligand. HA binds the cell surface receptor $\mathrm{CD} 44$, which is often overexpressed in tumor cells ${ }^{[140,141]}$. Hyaluronidases degrade HA; these enzymes are often highly abundant in the TME ${ }^{[142]}$.

The hyaluronic acid coat stimulated CD44-dependent cellular uptake of AuNSs. Upon digestion of hyaluronic acid, the targeting peptide localized the AuNSs to mitochondria. NIR-induced local PTT disrupted mitochondrial function, which decreased ATP production. As a result, drug efflux through P-gp, a process requiring ATP hydrolysis, was inhibited. At the same time, doxorubicin impaired cell survival. The combination of PTT and chemotherapy diminished the viability of SCC-7 squamous cell carcinoma cells, but was less effective in reducing tumor growth in SCC-7 mice. Importantly, the AuNSs enhanced doxorubicin retention in "MCF-7/ADR" cells, and AuNS/NIR treatment increased the survival of mice harboring "MCF-7/ADR" tumors. Collectively, these experiments showed that the AuNS-based "nanoheater" together with NIR irradiation decreased tumor cell proliferation in vitro and in mouse models. This reduction was observed for cancer cells with and without $\mathrm{MDR}^{[111]}$.

AuNCs defeated MDR in "MCF-7/ADR" cells through a combination of PTT and PDT ${ }^{[112]}$. The hollow interior of AuNCs was loaded with doxorubicin, quercetin and the phase-change material tetradecanol. AuNC cellular uptake was stimulated through the coating with PEGylated biotin. NIR illumination triggered AuNC-mediated localized heating which melted tetradecanol. Tetradecanol melting led to doxorubicin and quercetin release, which was accompanied by NIR-induced ROS production (PDT). For the AuNCs tested, several processes contributed to the death of "MCF-7/ADR" cells. Together, quercetin, ROS, and ATP-depletion diminished P-gp-dependent efflux of doxorubicin. The ensuing intracellular accumulation of doxorubicin, combined with ROS increase and ATP-depletion, promoted "MCF-7/ADR" cell apoptosis.

\section{Platinum nanoparticles}

Platinum nanoparticles (PtNPs) are characterized by extraordinary stability at extreme temperature ${ }^{[92]}$; their thermoplasmonic properties are ideal for photothermal therapeutics. Fucoidan is a natural bioactive polymer that can induce tumor cell apoptosis ${ }^{[183]}$. With "MCF-7/ADR" cells as model system, the impact of PtNPs coated with fucoidan and loaded with doxorubicin was examined ${ }^{[113]}$. These PtNPs were applied in a trimodal combination therapy (bio-thermo-chemo: fucoidan-PTT-doxorubicin), which integrated platinum-mediated PTT with two anticancer agents. The three branches of the treatment regimen worked synergistically and disrupted the MDR of "MCF-7/ADR" cells in vitro and in vivo. The possible underlying molecular mechanisms were identified. In particular, PtNP-based tumor cell elimination was accompanied by reduced signaling through the PI3-kinase/Akt/mTOR pathway, a route crucial for cancer cell survival and proliferation ${ }^{[184]}$.

\section{Palladium nanoparticles}

Palladium NPs (PdNPs) exhibit high photothermal conversion efficiency and photostability, which makes them excellent tools for $\mathrm{PTT}^{[163,164]}$. A recent study tested tetrapod-shaped copper-palladium alloy NPs (TNPs) for chemo-PTT, a combination of chemotherapy and $\mathrm{PTT}^{[114]}$. The copper-palladium alloy offers several benefits, such as tunable photothermal properties and copper-induced autophagy. Autophagy represents a major route for the degradation of proteins and organelles. In the context of cancer, autophagy 
can be cytoprotective or cytotoxic ${ }^{[185]}$. Chloroquine and 3-methyl adenine are established inhibitors of autophagy ${ }^{[185]}$.

Using copper-palladium alloy NPs, several cellular models confirmed the importance of NP morphology and composition for biomedical applications ${ }^{[114]}$. As such, the photothermal conversion efficiency of tetrapod-shaped PdNPs was greatly enhanced when compared to a spherical PdNP counterpart. Moreover, the copper content of the alloy NPs determined their ability to induce pro-survival autophagy. These features were explored for the killing of HeLa, 4T1 and "MCF-7/ADR" cells, and contributing mechanisms were uncovered. Specifically, NIR irradiation of cells containing TNP-1, a TNP with high copper content, elevated mitochondrial ROS production. The increase in ROS then triggered pro-survival autophagy. Under these conditions, pharmacological inhibition (3-methyl adenine, chloroquine) or genetic ablation (Atg5 knockout) of autophagy profoundly increased the cytotoxicity. The synergistic effect of TNP-1/NIR and the autophagy inhibitor 3-methyl adenine led to the efficient elimination of "MCF-7/ADR" cells. A synergistic effect of TNP-1-mediated PTT and autophagy inhibitors was also observed in "MCF-7/ADR" tumor-bearing NOD/SCID mice. Compared with control animals, PTT/autophagy inhibition decreased the tumor weight by $\sim 86 \%$ (3-methyl adenine) or $93 \%$ (chloroquine). Together, these experiments uncovered the potential of combined PTT and autophagy modulation for the killing of MDR and other cancer cells in vitro and in vivo.

\section{Lanthanide-doped upconverting nanoparticles}

Lanthanide-doped upconverting nanoparticles (UCNPs) absorb NIR light, which is converted via an anti-Stokes shift to light with higher energy. The light produced can be in the UV, visible, or NIR range of the spectrum ${ }^{[165,186]}$. UCNPs are especially interesting for biomedical research because their emission wavelength is tunable ${ }^{[187]}$. UCNPs have been evaluated for different modalities of cancer therapy, including the elimination of MDR cancer cells.

Several studies examined the effectiveness of UCNP-mediated PDT. To this end, different photosensitizers were conjugated to UCNPs and activated by NIR irradiation ${ }^{[15,131]}$. In one example, nanocomposites containing $\mathrm{TiO}_{2}$ as inorganic photosensitizer $\left(\mathrm{NaYF} 4: \mathrm{Yb} / \mathrm{Tm}-\mathrm{TiO}_{2}\right)$ were conjugated to folate as a cellular targeting moiety and then loaded with doxorubicin ${ }^{[15]}$. Low $\mathrm{pH}$ ( $\mathrm{pH}$ 6.0, $\mathrm{pH}$ 5.0) stimulated the release of doxorubicin, which was further increased by NIR irradiation. NIR light was also used for PDT, as it stimulated ROS generation by the photosensitizer $\mathrm{TiO}_{2}$. When compared with free doxorubicin, the nanocomposite/NIR treatment resulted in a significant loss of "MCF-7/ADR" cell viability in vitro as well as in vivo for "MCF-7/ADR" tumor-bearing mice ${ }^{[115]}$.

A multimodal UCNP nanoplatform was generated to overcome the cisplatin resistance of human ovarian cancer cells (A2780cisR) ${ }^{[131]}$. For this purpose, the UCNPs were conjugated to a platinum (IV) prodrug and functionalized with the photosensitizer Rose bengal (RB). These RB-Pt(IV)-UCNPs combined PDT with platinum-based chemotherapy. In brief, NIR irradiation of RB-Pt(IV)-UCNPs generated the visible light to activate Rose bengal and produce singlet oxygen $\left({ }^{1} \mathrm{O}_{2}\right)$. This led to an increase in cellular ROS levels, while delivery of the platinum (IV) prodrug facilitated genomic DNA crosslinking. The chemo-PDT combination treatment was toxic for cisplatin-sensitive (A2780) cells and their cisplatin-resistant counterparts (A2780cisR).

Another lanthanide-based approach was employed for combined chemo-PDT treatment ${ }^{[126]}$. Specifically, a lanthanide-doped silica framework containing europium and gadolinium oxides $\left(\mathrm{Eu}_{2} \mathrm{O}_{3}, \mathrm{Gd}_{2} \mathrm{O}_{3}\right)$ was loaded with doxorubicin. NIR-irradiation of these EuGdOx@MSF-DOX NPs produced singlet oxygen, diminished P-gp levels, and released doxorubicin. The chemo-PDT strategy profoundly reduced the viability of "MCF-7/ADR" cells ${ }^{[126]}$. 
Liposome/UCNP hybrid vesicles, called UCNP@Azo-Lipo/DOX, have been generated for the lightcontrolled release of doxorubicin ${ }^{[123]}$. The nanocarrier system is unique, as it addressed an important therapeutic issue - one-time burst $v s$. repeated cycles of drug delivery. UCNPs that emit UV and visible light upon NIR irradiation were key to control drug release and accomplish multiple delivery cycles.

Beginning with UCNPs, the delivery system was assembled in a stepwise fashion. A phospholipid monolayer was added to the UCNP surface to prevent particle agglomeration in aqueous environments. The UCNPs and doxorubicin were then co-encapsulated in artificial vesicles. The lipid bilayer of these vesicles contained azobenzene derivatives that undergo light-induced $c i s /$ trans isomerization ${ }^{[188]}$. UV light generates cis-isomers, which disrupts the lipid bilayer integrity and thereby promotes doxorubicin release from the vesicle. Visible light favors the formation of trans-isomers, which likely reseals the lipid bilayer. Using NIR-responsive UCNPs as the source of UV and visible light, the duration and intensity of NIR irradiation regulates drug release. Notably, multiple rounds of regulated drug release could be performed.

The system was tested in HeLa, MCF-7 and "MCF-7/ADR" cells in vitro; the combination of UCNP@AzoLipo/DOX and NIR treatment efficiently reduced the viability of all cell lines. Interestingly, UCNP@AzoLipo + NIR irradiation diminished the abundance of MRP1 (ABCC1) in "MCF-7/ADR" cells, which may re-sensitize cells to doxorubicin. Further evaluation of the delivery system was carried out with nude mice bearing MCF-7 or "MCF-7/ADR" derived tumors. (Folate was added to the lipid bilayer to improve tumor targeting.) Tumor growth was markedly reduced over a 2-week period, demonstrating that MDR could be overcome with the nanocarrier. Collectively, the study established a NIR-responsive delivery system that offers sophisticated control through repeated cycles of tunable drug release.

\section{Copper chalcogenides}

The chalcogens, or oxygen family of elements, include oxygen, sulfur, selenium, tellurium, and polonium. Metal oxide-based NPs are discussed in a separate section (see below). Due to their tunable localized surface plasmon resonance, copper sulfide $\left(\mathrm{Cu}_{2-\mathrm{x}} \mathrm{S}\right)$, copper selenide $\left(\mathrm{Cu}_{2-\mathrm{x}} \mathrm{Se}\right)$, and copper telluride $\left(\mathrm{Cu}_{2-\mathrm{x}} \mathrm{Te}\right)$ have favorable properties for NIR-based PTT and $\mathrm{PDT}^{[16-170,189,190]}$. In the context of medical applications, copper chalcogenides can resensitize chemoresistant tumor cells to drug treatment ${ }^{[16,136]}$. MDA-MB-453 breast cancer cells have high DNA methyltransferase (DNMT) activity and a mutation in the PTEN phosphatase gene; both features contribute to chemoresistance. However, a combinatorial approach based on doxorubicin-loaded $\mathrm{Cu}_{2-\mathrm{x}}$ Te nanocubes and NIR irradiation eliminated MDA-MB-453 cells in vitro ${ }^{[136]}$. With doxorubicin alone, the in vitro viability of MDA-MB-453 cells was largely unaffected ( $90 \%$ survival). By contrast, cell viability decreased to $11 \%$ when combined chemo-PTT-PDT therapy was performed with doxorubicin-loaded $\mathrm{Cu}_{2-\mathrm{x}}$ Te nanocubes. Due to the intrinsic ability of $\mathrm{Cu}_{2-\mathrm{x}}$ Te nanocubes to increase ROS production upon NIR illumination, the system is independent of extrinsic photosensitizers.

Another study treated "MCF-7/ADR" cells with a multifunctional CuS-based nanostructure ${ }^{[116]}$. When irradiated with NIR light, the NPs released NO, and doxorubicin accumulated in cells. This was accompanied by a small decrease in P-gp abundance and a $30 \%$ loss in the viability of "MCF-7/ADR" cells compared with untreated controls.

\section{Transition metal dichalcogenides}

Two-dimensional transition metal dichalcogenides (2D TMDCs) are sandwich-like nanostructures, composed of a central layer of transition metal, surrounded by a layer of chalcogenides on each side ${ }^{[191,192]}$. TMDCs are generally described as $\mathrm{MX}_{2}$, with $\mathrm{M}$ representing the transition metal (group 4-10 in the periodic table) and $\mathrm{X}$ the chalcogen $(\mathrm{S}, \mathrm{Se}, \text { or Te })^{[193]}$. TMDCs display strong NIR absorption, tunable optical properties, and a large surface area available for modification. Accordingly, TMDCs are suitable nanomaterials for PTT. When combined with conventional chemotherapy, the PTT or PDT elicited by various TMDCs (e.g., $\mathrm{MoS}_{2}^{[194]}, \mathrm{MoSe}_{2}^{[195-197]}$, $\mathrm{WS}_{2}{ }^{[198]}$ ) efficiently eliminated tumor cells. 
The TMDC-based multifunctional nanoplatform $\mathrm{MoS}_{2}-\mathrm{PEI}-\mathrm{HA}^{[117]}$ carries hyaluronic acid (HA) for active targeting to the tumor site. To achieve localized drug release, the nanomaterial takes advantage of HA degradation by hyaluronidases. These enzymes are often highly abundant in tumors and the TME ${ }^{[142,199]}$. Combined with NIR irradiation, Dox@MoS - -PEI-HA (the doxorubicin-loaded version of $\mathrm{MoS}_{2}$-PEI-HA) significantly reduced "MCF-7/ADR" viability in vitro when compared with doxorubicin or Dox@MoS PEI-HA alone ${ }^{[117]}$. Several mechanisms likely contributed to "MCF-7/ADR" elimination, such as increased doxorubicin accumulation, heat production, and diminished P-gp abundance. The Dox@MoS -PEI-HA/ NIR combination was also effective for the treatment of "MCF-7/ADR" tumor-bearing mice. At day 25 of the experiment, the multimodal therapy prevented tumor growth by $\sim 96 \%$. Moreover, no tumor recurrence was observed, which was detected in all other experimental groups at day 14 or earlier.

\section{Metal oxides}

Metal oxides have been widely studied in cancer research; specific examples include iron ${ }^{[200-202]}$, $\operatorname{titanium}^{[203-205]}$, and tungsten oxides ${ }^{[206-208]}$. Iron oxides are of therapeutic interest because they absorb the energy of the magnetic field to produce hyperthermia, which can be applied for the killing of cancer cells $^{[70,209,210]}$.

The photothermal effect of metal oxides can also be used to release NO from a heat-sensitive NO donor $(\mathrm{SNO})^{[118]}$. To this end, $\mathrm{Fe}_{3} \mathrm{O}_{4} @$ polydopamine@mesoporous silica NPs were coupled to transferrin for cellular targeting and to SNO for NO production. These phototriggered NO nanogenerators (PTNGs) were loaded with doxorubicin and assessed in "MCF-7/ADR" cells in vitro and in "MCF-7/ADR" tumorbearing BALB/c nude mice. Notably, when PTNGs were combined with NIR irradiation, the abundance of P-gp was significantly reduced in "MCF-7/ADR" cells. At the same time, the intracellular concentration of doxorubicin increased markedly. Moreover, the combination doxorubicin-loaded PTNGs/NIR profoundly diminished the viability of "MCF-7/ADR" cells when compared with doxorubicin-loaded PTNGs alone. These results support the idea that NO contributed to the therapeutic effects of PTNGs. In vivo studies confirmed this interpretation, as the combination treatment doxorubicin-loaded PTNGs/NIR led to a marked reduction of tumor volume and size in experimental mice.

To develop new tools for the elimination of drug-resistant cancer cells, iron/gold NPs were incorporated in porous silicon particles ${ }^{[157]}$. The nanocomposites were super-paramagnetic and could perform multiple cycles of photothermal conversion. Aside from NIR light, the NPs responded to additional external stimuli. For instance, application of an electric field improved the uptake of nanocomposites, both for $2 \mathrm{D}$ and spheroid cultures of "MCF-7/ADR" cells. The increase in cellular uptake may be explained by the formation of micro-aggregates or the accumulation of nanocomposites on the cell surface. The latter process is commonly employed for drug delivery or magnetofection ${ }^{[211]}$.

When nanocomposites were loaded with doxorubicin, drug release was stimulated by lowering the $\mathrm{pH}$ from 7.4 to 5.4; the release was further increased by NIR irradiation. The highest level of drug accumulation in nuclei was observed when "MCF-7/ADR" cells were exposed to doxorubicin-loaded nanocomposites, an external magnetic field, and NIR irradiation. By contrast, the combination free doxorubicin/external magnetic field/NIR was ineffective to concentrate the drug in nuclei. The same results were obtained for "MCF-7/ADR"-derived spheroids. In summary, the study developed an iron/gold-containing nanocomposite that responded to several external stimuli and provided a tool to concentrate doxorubicin in the nuclei of MDR cancer cells.

\section{Carbon-based nanoparticles}

Carbon nanomaterials are noticeable for their structural diversity, optical features, high biocompatibility and -in general- low cytotoxicity ${ }^{[212,213]}$. Nevertheless, carbon nanomaterials can modulate the immune 
system; depending on the particle features, immune reactions were activated or suppressed ${ }^{[214]}$. Carbon nanotubes, carbon nanospheres, graphene, graphene oxides, and fullerenes represent major classes of carbon-based NPs. Some of these nanomaterials are NIR-responsive and characterized by high photothermal conversion ${ }^{[215-217]}$. Due to their unique properties, carbon-based NPs are suitable for PTT or $\mathrm{PDT}^{[212,218,219]}$. Carbon nanotubes are the most frequently used carbon-based NPs that have been thoroughly examined for drug delivery ${ }^{[220]}$. They are characterized by excellent mechanical properties, which combine high rigidity with good flexibility ${ }^{[21]}$. The elongated morphology of carbon nanotubes likely facilitates the translocation across biological membranes ${ }^{[222]}$. At the same time, this shape may play a part in the cytotoxicity of carbon nanotubes ${ }^{[214]}$. By contrast, carbon nanospheres are less likely to cause physical damage to living cells ${ }^{[124]}$. Graphene oxide has several advantages for biological applications; it is stable, yet flexible, dispersible in aqueous biological environments and easily amenable to modification ${ }^{[213,221]}$.

\section{Carbon nanotubes}

Single-walled carbon nanotubes offer high NIR absorbance and large surfaces for drug loading ${ }^{[223]}$. These attributes were explored to treat chemoresistant human leukemia K562R cells ${ }^{[138]}$. K562R cells have elevated levels of P-gp, which is located in the plasma membrane ${ }^{[139]}$. To improve targeting to K562 R cells, the carbon nanotubes were functionalized with an antibody against P-gp. Indeed, the antibody enhanced binding of carbon nanotubes to K562R cells, but not to their drug-sensitive counterpart (K562S cells). To accomplish drug delivery, the single-walled carbon nanotubes were loaded with doxorubicin, which was efficiently released upon NIR irradiation. Taken together, the study showed that single-walled carbon nanotubes (conjugated to anti-P-gp antibodies and loaded with doxorubicin) combined with NIR irradiation significantly reduced the viability of K562R cells in vitro. Hence, functionalized single-walled carbon nanotubes can overcome MDR, which is ascribed - at least in part - to the increased biosynthesis of P-gp ${ }^{[139]}$.

To combat the MDR of "MCF-7/ADR" cells, single-walled nanotubes were modified with PEGylated phospholipids and loaded with doxorubicin ${ }^{[125]}$. Following cellular uptake of the carbon nanotubes and NIR illumination, doxorubicin accumulated in cell nuclei. This approach caused a significant loss of "MCF7/ADR" cell viability when compared with non-irradiated control samples.

\section{Carbon nanospheres}

Hollow mesoporous carbon nanospheres can operate as drug carriers, while also providing PTT and PDT modalities. Together, these functionalities can combat the MDR of "MCF-7/ADR" cells ${ }^{[124]}$. Thus, NIR irradiation of doxorubicin-loaded carbon nanospheres initiated several processes that are relevant to cancer therapy. While heat, singlet oxygen, and ROS were produced, the intracellular concentration of doxorubicin increased. In addition, HSF-1 accumulated in nuclear foci, and the protein abundance of P-gp and p53 was reduced. Treatment with doxorubicin-loaded carbon nanospheres and NIR irradiation had synergistic effects, which cumulated in a significant loss of "MCF-7/ADR" cell viability in vitro.

\section{Graphene oxides}

The expression of several genes encoding drug efflux pumps is high in human MDA-MB-231 breast cancer cells $^{[134]}$. To reduce the associated drug resistance, a chemo-PTT strategy with two therapeutic drugs was developed ${ }^{[133]}$. For this purpose, graphene was stabilized with poloxamer 188, a compound that also inhibits efflux pumps ${ }^{[153]}$. The nanocarrier was loaded with doxorubicin and irinotecan and then assessed for the killing of MDA-MB-231 cells. The dual drug-loaded graphene oxide increased the abundance of cyclin-dependent kinase inhibitors (p21, p27) and the tumor suppressor p53. These changes are consistent with an upregulation of cell apoptosis ${ }^{[133]}$. However, the effect may be independent of the nanocarrier, as the simultaneous incubation with doxorubicin and irinotecan led to the same changes in p21, p27 and p53 abundance. Nevertheless, the combination dual drug-loaded nanocarrier/NIR led to a profound reduction of tumor cell viability. 
Another nanocarrier based on graphene oxide was developed for chemo-PTT of "MCF-7/ADR" cells ${ }^{[119]}$. NP surface engineering was aligned to the $\mathrm{pH}$ differences that NPs encounter during circulation, in the TME, and when located in cellular endosomes/lysosomes. Specifically, the graphene oxide carrier was coated with two different polymers. One of these polymers was further modified to provide $\mathrm{pH}$-responsiveness. Following loading with doxorubicin, the $\mathrm{pH}$-dependent changes of the nanocarrier had two effects that are pertinent to future in vivo applications. First, the overall positive charge of the delivery system at $\mathrm{pH}$ 6.8, which mimicked the TME, could facilitate uptake by tumor cells. Second, relevant to the lysosomal interior, doxorubicin release was stimulated at $\mathrm{pH}$ 5.0. Doxorubicin-loaded $\mathrm{pH}$-responsive nanocarriers reduced the viability of "MCF-7/ADR" cells, which was further diminished by NIR irradiation. The effect of both treatment modalities was synergistic. Incubation with free doxorubicin or NIR irradiation alone led to only minor loss of cell viability. The two examples in this section demonstrate that the co-application of drug-loaded graphene oxide NPs and NIR light is suitable to overcome MDR in different in vitro models.

\section{Polydopamine}

Due to their high photothermal conversion efficiency, polydopamine (PDA) and other polymers have been used as photothermal agents ${ }^{[91]}$. Polydopamine stands out because of its unique chemical properties, adherence to numerous surfaces, biocompatibility, high photoconversion rate, and ability to co-deliver multiple drugs ${ }^{[224,225]}$. Given their notable intrinsic PTT capability, a range of polydopamine-based structures (colloidal, hollow, and composite particles) have been assessed as NIR-responsive theranostic tools $^{[225]}$. For example, polydopamine-containing nanostructures were examined in MDR cancer cells ${ }^{[127-129]}$.

Recent work exploited the polydopamine-mediated photothermal effect to release doxorubicin and generate NO. NO production was part of a triple treatment modality to surmount the MDR of "MCF-7/ADR" cells in vitro and in vivo. This was achieved with a polydopamine-based nanocomposite, which carried a NO donor and was loaded with doxorubicin ${ }^{[127]}$. NIR illumination triggered polydopamine-mediated PTT, $\mathrm{NO}$ and doxorubicin release. This triple combination of chemo-PTT and NO production diminished the viability of "MCF-7/ADR" cells and was associated with a marked reduction of P-gp abundance. Moreover, the triple combination strategy profoundly diminished the tumor volume in "MCF-7/ADR" tumor-bearing mice at treatment day 30 .

Another study also applied a triple combination therapy to overcome MDR in "MCF-7/ADR" cells ${ }^{[128]}$. The approach used two different NIR-sensitive polydopamine NPs. One NP type could generate NO; the second was loaded with doxorubicin for chemo-PTT. Co-incubation with both NP classes and subsequent NIR irradiation caused a marked loss of "MCF-7/ADR" cell viability. The exposure to NO-producing NPs/NIR achieved important therapeutic goals that are relevant to MDR. Thus, the abundance of ABC efflux pumps was reduced. Simultaneously, intracellular ATP was depleted, which further diminished drug efflux.

The in vitro experiments were expanded to "MCF-7/ADR"-bearing mice. Successive treatment with (1) NO-producing NPs/NIR, and (2) doxorubicin-loaded NPs/NIR reduced the tumor volume profoundly. At the same time, caspase-3 abundance was increased, whereas Bcl-2 levels were reduced. These changes are consistent with increased tumor cell apoptosis in NP-treated animals when compared with control animals that received the vehicle PBS.

A polydopamine-based nanoplatform was also developed for chemo-PTT ${ }^{[129]}$. In particular, the microtubule-stabilizing drug docetaxel was loaded onto a biodegradable polymer, and then encapsulated by a polydopamine shell. The resulting NP was further modified with the P-gp inhibitor TPGS [Table 2]. The release of docetaxel from the nanoplatform was facilitated by lowering the $\mathrm{pH}$ from 7.4 to 5.0 and NIR irradiation. In vitro, the nanoplatform/NIR combination reduced the viability of "MCF-7/ADR" cells; the loss of viability was higher when compared with free docetaxel. In a mouse model, "MCF-7/ADR" tumor 
weight and volume were significantly decreased relative to the docetaxel-treated animals at day 14 of the treatment period. Collectively, the study demonstrated that polydopamine-based NPs are appropriate for chemo-PTT that delivers docetaxel and inhibits P-gp.

\section{Black phosphorus}

The exploration of black phosphorus for biomedical applications, especially as an alternative to graphenerelated materials, has begun only recently, and many hurdles remain ${ }^{[226]}$. Nevertheless, black phosphorus is relevant to cancer theranostics because it is biocompatible, biodegradable, and suitable for multiple applications. Examples are imaging, biosensing, drug delivery, PTT, PDT, sonotherapy, and the combination with other modalities, such as immunotherapy or cancer starvation therapy ${ }^{[27-232]}$. Key to the appeal of black phosphorus is its ability to form two-dimensional layered sheets. Importantly, the number of layers in a sheet can be controlled during fabrication. As a larger number of layers narrow the bandgap, the absorption properties of black phosphorus are tunable over the UV, visible, and NIR range of the light spectrum $^{[226,229,233]}$. Although black phosphorus is biodegradable and degradation products are non-toxic, the material has notable disadvantages. Black phosphorous material has limited stability in the presence of oxygen or in aqueous solutions ${ }^{[226]}$. Nevertheless, capping layers or chemical surface modifications can protect black phosphorous nanosheets in biologically relevant environments. Moreover, black phosphorous nanosheets, in conjunction with NIR-irradiation, have been used to counteract the chemoresistance of cancer cells ${ }^{[120,121,132]}$. This suggests that health-related applications based on black phosphorous nanomaterials are feasible in the future. Several recent publications support this optimism.

Polydopamine-coated black phosphorous nanosheets provided the platform for a combined gene/ chemo-PTT approach to defeat cancer drug resistance ${ }^{[120]}$. The strategy was based on black phosphorous nanosheets that were functionalized and modified. Specifically, for P-gp gene knockdown siRNA was incorporated into the nanosheets, which were also loaded with doxorubicin. Association with the nanosheet protected P-gp siRNA against RNase-mediated degradation. Finally, a protective polydopamine film enhanced the stability and photothermal capacity of the nanosheets, and the DNA aptamer AS1411 was surface-conjugated for cancer cell targeting. The resulting nanosheets were assessed for gene/chemoPTT.

Several parameters of the nanosheets controlled doxorubicin release, $\mathrm{pH}$ (discharge elevated at $\mathrm{pH} 5.0$ as compared to $\mathrm{pH}$ 7.4), the polydopamine coat, and the NIR-triggered photothermal effect. Interestingly, NIR irradiation promoted the breakdown of the polydopamine coat and the black phosphorous nanosheet.

When tested in "MCF-7/ADR" cells, the highest toxicity was accomplished for the gene/chemo-PTT combination, whereas the simultaneous treatment with doxorubicin and P-gp siRNA had little impact. The effectiveness of the black phosphorous-based nanosheets was confirmed with "MCF-7/ADR" tumorbearing nude mice in vivo. On treatment day 20, the targeted (AS411) gene/chemo-PTT approach led to a significant reduction of tumor volume relative to free doxorubicin/siRNA. Concurrently, P-gp abundance was markedly reduced in tumor cells.

A different study challenged cancer cell MDR with black phosphorous nanosheets that were designed for chemo-PTT-PDT. The three-pronged approach required NIR irradiation ${ }^{[121]}$. To this end, black phosphorous nanosheets were loaded with doxorubicin and protected by polydopamine. Finally, the nanosheets were modified with phenethyl isothiocyanate, a natural compound produced by cruciferous vegetables. Phenethyl isothiocyanate is relevant because it decreases the misfolding of mutant p53 protein ${ }^{[234]}$. (Note: the TP53 gene is often mutated in tumor cells, and mutant p53 can promote cancer cell survival.) The chemo-PTT-PDT protocol was successful to overcome cancer cell MDR in vitro and in vivo. In particular, the triple combination treatment resulted in the lowest "MCF-7/ADR" cell viability when compared with 
control and other treatment groups. This outcome was verified in vivo with "MCF-7/ADR" tumor-bearing mice, as the smallest tumor volume was obtained with the chemo-PTT-PDT experimental group.

Another black phosphorous-based nanocarrier was examined with cisplatin-resistant human ovarian cancer cells (A2780cisR), hepatocellular carcinoma cells (HepG2cisR), their non-resistant counterparts (A2780cisN, HepG2cisN), and non-cancer cell lines ${ }^{[132]}$. For this study, the stability of black phosphorous nanosheets was improved by surface coordination with a cisplatin derivative. The PTT capability was preserved in these stabilized nanosheets. The nanosheets also elicited a DNA damage response in HepG2cisN and HepG2cisR cells, which was enhanced by NIR irradiation. Interestingly, the in vitro toxicity of the drug-modified black phosphorous nanosheets was higher for some cancer cell lines than for the non-tumor cell lines studied. In the future, these differences could potentially be exploited for the selective killing of tumor cells in vivo.

\section{Other NIR-responsive materials for the elimination of multidrug resistant cancer cells}

This review presents NIR-responsive nanocarriers for the eradication of drug-resistant cancer cells. Our focus is on nanomaterials that are based on metals, carbon, polydopamine, or black phosphorus. Protocols that rely on other NIR-excitable agents are not discussed. However, it should be noted that light-responsive vesicles, micelles, and small molecule nanoassemblies continue to be assessed for cancer theranostics ${ }^{[235-237]}$.

\section{LIMITATIONS OF NIR-RESPONSIVE NANOMATERIALS TO DEFEAT CANCER DRUG RESISTANCE AND FUTURE DIRECTIONS}

Despite great promise of the NIR-responsive NPs discussed here, technical and other restrictions have so far prevented their wide-spread use for the improvement of human health ${ }^{[51]}$. The current shortcomings are related to diverse issues, ranging from NP synthesis to the optimal choice of molecular targets for NPbased therapies. To generate clinically relevant treatment regimens with NIR-responsive NPs in the future, multidisciplinary solutions are mandatory. They have to be accompanied by rigorous evaluation of NP properties and performance, from the initial synthesis to clinical trials [Figure 3]. Table 5 summarizes the topics that will benefit from further research and development.

\section{Stimulus-responsive NPs}

Synthesis and quality control

As discussed for theranostic nanomaterials in general ${ }^{[57]}$, the fabrication and quality control of NIRresponsive NPs are often complex, and large-scale synthesis is not a trivial task ${ }^{[239,240]}$. Yet, simple production protocols with low batch-to-batch variability and good manufacturing practices are necessary for the transition to clinical trials ${ }^{[241]}$. These features, and ideally low costs, are pre-requisites for health application and commercialization; they are frequently neglected in basic research.

\section{Nanomaterial properties}

Besides the constraints imposed by tumor location and vulnerability of healthy tissues, the nanomaterial properties also determine the effectiveness of NIR irradiation. In particular, the photoconversion efficiency of NPs has to be matched to the treatment modality. Thus, low quantum yield of upconverting nanomaterials will be inadequate for applications that rely on high photon upconversion ${ }^{[242]}$.

\section{Adding new features to NIR-responsive NPs}

Although simple and reliable protocols are compulsory for NP synthesis, new attributes could enhance their in vivo performance. In particular, relatively unexplored for the applications discussed here is the incorporation of phase-change materials. Recent studies demonstrate the potential of tetradecanol for cancer theranostics ${ }^{[112,243,244]}$ and warrant further assessment for NP-based approaches. 


$\begin{array}{ll}\text { QIR-responsive } & \text { 2D cell culture }\end{array}$

Figure 3. Important parameters for the evaluation of near-infrared (NIR)-responsive nanoparticles. The proper design, synthesis and evaluation of NIR-responsive nanoparticles (NPs) require methodical and quantitative assessment at different stages. Beginning with optimization of the synthesis, NPs are evaluated in $2 \mathrm{D}$ and $3 \mathrm{D}$ cell cultures, as well as in pre-clinical animal models ${ }^{[253]}$. If NPs perform adequately at these stages, they may progress to clinical trials. The figure depicts some of the critical readouts for each level of evaluation. See main text for additional details

\section{Table 5. Factors and pathways involved in cancer MDR}

\begin{tabular}{|c|c|}
\hline Factor, pathway & Future studies related to stimuli-responsive NPs and multidrug resistant cancer \\
\hline Long-term NP toxicity & $\begin{array}{l}\text { Evaluation of NP toxicity after long-term exposure or repeated treatment cycles in appropriate in } \\
\text { vivo models }\end{array}$ \\
\hline NP clearance & Appropriate NP clearance, especially for multiple rounds of treatment \\
\hline Combination therapy & $\begin{array}{l}\text { Evaluation of efficacy for NP-based and other treatment modalities; } \\
\text { whole body pharmacokinetics; } \\
\text { assessment of synergistic effects for multi-modal therapy }\end{array}$ \\
\hline Different drug transporters & Explore antibodies for NP targeting and transporter inhibition \\
\hline Other factors promoting MDR & Gene mutations, epigenetic changes, cell signaling \\
\hline Cancer stem cells (CSC) & Increase CSC vulnerability to NP-based treatment \\
\hline Tumor microenvironment & Tumor vasculature, immune and other cells in TME, non-cellular components of TME \\
\hline Immune system & Harness or enhance immune functions to eliminate MDR cancer cells \\
\hline Stress resistance & $\mathrm{NF}-\kappa \mathrm{B}, \mathrm{HSF}-1$, molecular chaperones \\
\hline Cancer cell and TME metabolism & Hypoxia, autophagy, other metabolic changes related to cancer cell survival \\
\hline microRNAs & Regulation of tumor cell survival \\
\hline Computational and mathematical modeling & Predict outcomes of NP-based regimens and test models in vitro and in vivo \\
\hline Non-druggable targets & Silencing strategies, gene editing \\
\hline
\end{tabular}

Near-infrared (NIR)-responsive nanoparticles (NPs) impact various components and activities, which are often not fully characterized. Alternative and complementary strategies are proposed that may enhance the efficacy of NIR-responsive NPs for the treatment of chemo-resistant cancers. Additional details are provided in the main text. MDR: multidrug resistance; TME: tumor microenvironment

\section{NIR irradiation}

Selection of optimal NIR wavelength

Specific aspects of NIR-responsive NPs deserve further attention to improve their performance related to cancer MDR. The advantage of NIR light over visible or UV light for tumor irradiation is clearly 
established. Nevertheless, the NIR penetration depth is still limited; it depends on the tumor location, light intensity and wavelength ${ }^{[82]}$. Hence, inadequate penetration depth may preclude efficient NIR-induced drug release at some tumor sites ${ }^{[165]}$. Compared to the widely examined NIR-I window $(700-900 \mathrm{~nm})$, the NIR-II window (1,300-1,700 nm) offers enhanced tissue penetration ${ }^{[80]}$. (Note: the demarcation of NIR windows varies among publications.) Accordingly, NIR-II-responsive nanomaterials may be superior for the detection of small primary tumors and metastases ${ }^{[245,246]}$.

\section{Effects on healthy tissue}

Although generally considered safe, high-intensity NIR light, prolonged or repeated cycles of NIR exposure may have negative impact on healthy cells. This includes tissue overheating, membrane re-organization, neural stimulation, mitochondrial ROS production, mitochondrial DNA damage, and changes in gene expression $^{[82,247,248]}$. While these side effects could be tolerable for cancer theranostics, they may have to be considered if treatment regimens require repeated rounds of NIR irradiation.

\section{Nano-safety}

A major concern related to in vivo applications of nanomaterials is long-term safety. This applies especially to nanocarriers that are not biodegradable, could leach toxic components, or accumulate in various tissues and organs. This concern is well documented for metal- and carbon-based NPs, as they may concentrate in the liver, lung, and spleen, with potentially deleterious consequences for organ functions ${ }^{[249-251]}$.

In vitro models often provide information on the toxicity and mechanism-of-action. Nonetheless, in vivo studies are required to define the organismal toxicity, whole body pharmacokinetics, and immune responses related to nanomaterials ${ }^{[252]}$ [Figure 3]. Rodents, especially tumor-bearing mice, are the most common experimental animals for this research. However, many aspects of mouse models have only limited clinical relevance; their specific shortcomings have been discussed previously ${ }^{[57]}$.

\section{Bio-nano interactions}

The European Union has developed guidelines for the characterization and evaluation of UCNPs ${ }^{[187]}$. In addition, recommendations for the preclinical assessment of nanomaterials are available ${ }^{[253]}$. Many of these recommendations also apply to the NIR-responsive nanomaterials discussed here. Mathematical and computational modeling approaches could provide additional guidance to the field ${ }^{[254]}$.

A surprising lack of insight into even basic aspects of bio-nano interactions impedes progress in the field and holds back clinical translation. The complexities of these interactions are only emerging. For example, the role of NP elasticity for cellular uptake has only recently been demonstrated ${ }^{[255]}$ and remains to be explored for the treatment of chemo-resistant cancer.

Remarkably, the pharmacokinetics and pharmacodynamics of NP-based applications, especially as they relate to MDR, are frequently poorly defined ${ }^{[2]}$. This applies in particular to whole-body pharmacokinetics of the stimulus-responsive NPs discussed here. Many studies combine multiple treatment modalities and claim synergistic effects. However, an appropriate quantitative calculation of synergy is rarely provided in published work ${ }^{[256]}$. Furthermore, whether biocompatible doses of the NP/NIR combination surmount MDR often remains to be determined. A rigorous understanding of these parameters is compulsory to propel the field towards clinically relevant contributions.

Current limitations are further illustrated by the focus on strategies that target drug efflux pumps and employ NPs for the direct cancer cell killing. By contrast, the NP-based modulation of other targets and especially cancer support systems remain to be further explored (see below). Thus, several key issues require attention to uncover the full potential of NIR-responsive NPs for cancer therapy. 


\section{New potential targets for NIR-responsive NPs}

Diverse causes of cancer drug resistance

In the context of MDR, nanomedicine has largely focused on $\mathrm{ABC}$ transporters. However, multiple biological mechanisms advance cancer MDR in human patients. Inclusion of MDR-relevant factors that are mostly ignored will expand the potential of therapeutic NPs. The epigenetic machinery, such as DNA methyl transferases (see above), provides possible targets for further research ${ }^{[257]}$.

\section{Cancer stem cells, the tumor microenvironment, and immune responses}

CSCs and the TME are major contributors to MDR and cancer-related patient death ${ }^{[2,4,10,11]}$. So far, NIRabsorbing NPs have been evaluated predominantly for the killing of drug-resistant proliferating tumor cells. Thus, including CSCs as well as the cellular and non-cellular constituents of the TME will broaden the range of NP applications. This may involve the development of NPs that can prevent -or reverse- CSC dormancy.

In the TME, tumor macrophages, metalloproteases, and the tumor vasculature are potential targets to offset MDR. NIR-sensitive upconverting nanocrystals that reprogram tumor-associated macrophages or aim at metalloproteases exemplify this strategy ${ }^{[258,259]}$. Emerging mechanisms of NP extravasation from endothelial cells highlight the importance of matching NP properties to the biological constraints of the $\mathrm{TME}^{[59,260]}$. In this scenario, improving transcytosis across endothelial cells could increase NP concentrations in the tumor. As well, appropriate NP surface modifications could achieve this task ${ }^{[260]}$. Interestingly, PDT delivered by NIR-responsive UCNPs can augment the permeability of tumor blood vessels, thereby promoting NP extravasation into the tumor ${ }^{[261]}$. The same outcome is accomplished by impairing the endothelial barrier function with magnetic nanomaterials ${ }^{[262,263]}$.

There has been immense effort to identify -and prevent- the immune responses elicited by $\mathrm{NPs}^{[264]}$. Yet, alternative approaches are also being considered. In particular, NPs could modulate the activities of innate and adaptive immune systems towards the ultimate goal of eliminating cancer cells ${ }^{[57,265,266]}$. As NIR photoimmunotherapy can activate immune functions directed against cancer cells ${ }^{[267]}$, the modality seems especially suited to enhance the performance of NIR-responsive NPs.

\section{Cancer cell metabolism}

Cancer cells and the TME display prominent metabolic and signaling alterations ${ }^{[268]}$. These pathological changes, exemplified by hypoxia and the increased abundance of glutathione, foster treatment resistance ${ }^{[269,270]}$. The rewiring of cancer cell metabolism offers a plethora of therapeutic opportunities that can be addressed with NIR-responsive NPs.

\section{Non-druggable contributors to cancer multidrug resistance}

Until now, strategies to overcome cancer MDR with NIR-responsive NPs have largely disregarded the possibility to target non-druggable components. Thus, NPs that deliver siRNAs or oligonucleotides ${ }^{[271]}$ to cancer cells and the TME could open new opportunities for nanomedicine. Furthermore, gold nanoclusters or AuNPs have successfully delivered components of the CRISP/Cas9 system for gene editing to cancer cells $^{[272,273]}$. Future studies will determine to which extent this emerging methodology is suitable to counteract cancer cell MDR.

\section{Clinical translation}

\section{Clinical trials}

To date, only a small number of cancer-related clinical trials have been carried out with the nanomaterials described in this review. Indeed, the vast majority of NPs that are currently in clinical trials are liposomebased $^{[274]}$. A notable exception is AuroLase therapy, which uses AuroShells ${ }^{\circledR}$, NIR-responsive gold-silica nanoshells, for the thermo-ablation of tumor cells. AuroLase therapy has been evaluated in clinical trials 
for lung, head-and-neck, as well as prostate cancer ${ }^{[275]}$. Promising treatment outcomes have been reported for prostate cancer ${ }^{[276]}$. Moreover, several magnetic NPs are FDA-approved for magnetic hyperthermia, which can be enhanced by NIR irradiation ${ }^{[209,277]}$.

\section{Future directions}

The potential of NIR-responsive NPs for cancer therapy has been widely emphasized. However, their specific role in therapy is not always clear. Many cancer patients receive standard treatment that consists of surgery, radiotherapy, chemotherapy, immunotherapy or combinations thereof. Combination treatments that eradicate MDR cancer cells by targeting different resistance pathways are particularly efficient. Consequently, appropriate NP-based approaches must be selected to complement standard or other treatment regimens.

\section{CONCLUSION}

Taken together, there has been remarkable advancement in the design, synthesis and evaluation of NIRresponsive nanomaterials in vitro and in animal models of cancer cell MDR. At the same time, translation to the clinic has been rare. To achieve this transition, future studies will have to answer a wide variety of questions to ensure the successful treatment of human patients (examples in Figure 3). The topics range from basic physiology, such as NP-triggered immune responses, to the development of appropriate treatment regimens, as exemplified by the route of NP administration and dosage. In the long run, the ability to improve patient survival and/or quality of life will determine the success of NIR-responsive NPs in the clinic.

\section{DECLARATIONS}

\section{Authors' contributions}

Participated in study design, drafted, and wrote the manuscript: Chu S, Stochaj U

Read and approved final version of the manuscript: Chu S, Stochaj U

\section{Availability of data and materials}

Not applicable.

\section{Financial support and sponsorship}

Natural Sciences and Engineering Council of Canada (to Stochaj U) and Mitacs fellowship (to Chu S).

\section{Conflicts of interest}

The authors declared that there are no conflicts of interest.

\section{Ethical approval and consent to participate}

Not applicable.

\section{Consent for publication}

Not applicable.

\section{Copyright}

(c) The Author(s) 2020.

\section{REFERENCES}

1. Gotwals P, Cameron S, Cipolletta D, Cremasco V, Crystal A, et al. Prospects for combining targeted and conventional cancer therapy with immunotherapy. Nat Rev Cancer 2017;17:286-301.

2. Konieczkowski DJ, Johannessen CM, Garraway LA. A convergence-based framework for cancer drug resistance. Cancer Cell 
2018;33:801-15

3. Kathawala RJ, Gupta P, Ashby CR Jr, Chen ZS. The modulation of ABC transporter-mediated multidrug resistance in cancer: a review of the past decade. Drug Resist Updat 2015;18:1-17.

4. Holohan C, Van Schaeybroeck S, Longley DB, Johnston PG. Cancer drug resistance: an evolving paradigm. Nat Rev Cancer 2013;13:714-26.

5. Gray R, Bradley R, Braybrooke J, Liu Z, Peto R, et al. Increasing the dose intensity of chemotherapy by more frequent administration or sequential scheduling: a patient-level meta-analysis of 37298 women with early breast cancer in 26 randomised trials. The Lancet 2019;393:1440-52.

6. Smorodinsky-Atias K, Soudah N, Engelberg D. Mutations that confer drug-resistance, oncogenicity and intrinsic activity on the ERK MAP kinases — current state of the art. Cells 2020;9:E129.

7. Bell CC, Gilan O. Principles and mechanisms of non-genetic resistance in cancer. Br J Cancer 2019;122:465-72.

8. Sridharan S, Howard CM, Tilley AMC, Subramaniyan B, Tiwari AK, et al. Novel and alternative targets against breast cancer stemness to combat chemoresistance. Front Oncol 2019;9:1003: 1-19.

9. Vasan N, Baselga J, Hyman DM. A view on drug resistance in cancer. Nature 2019;575:299-309.

10. Eramo A, Ricci-Vitiani L, Zeuner A, Pallini R, Lotti F, et al. Chemotherapy resistance of glioblastoma stem cells. Cell Death Differ 2006;13:1238-41.

11. Begicevic RR, Falasca M. ABC Transporters in cancer stem cells: beyond chemoresistance. Int J Mol Sci 2017;18:E2362.

12. Holohan C, Van Schaeybroeck S, Longley DB, Johnston PG. Cancer drug resistance: an evolving paradigm. Nature Reviews Cancer 2013;13:714-26.

13. Heryanto YD, Achmad A, Taketomi-Takahashi A, Tsushima Y. In vivo molecular imaging of cancer stem cells. Am J Nucl Med Mol Imaging 2015;5:14-26.

14. Gao J, Feng SS, Guo Y. Nanomedicine against multidrug resistance in cancer treatment. Nanomedicine 2012;7:465-8.

15. Steinbichler TB, Dudas J, Skvortsov S, Ganswindt U, Riechelmann H, et al. Therapy resistance mediated by cancer stem cells. Semin Cancer Biol 2018;53:156-67.

16. Assaraf YG, Brozovic A, Gonçalves AC, Jurkovicova D, Linē A, et al. The multi-factorial nature of clinical multidrug resistance in cancer. Drug Resistance Updates 2019;46:100645.

17. Amawi H, Sim HM, Tiwari AK, Ambudkar SV, Shukla S. ABC transporter-mediated multidrug-resistant cancer. Adv Exp Med Biol 2019;1141:549-80.

18. Robey RW, Pluchino KM, Hall MD, Fojo AT, Bates SE, et al. Revisiting the role of ABC transporters in multidrug-resistant cancer. Nature Reviews Cancer 2018;18:452-64.

19. Shukla S, Chen ZS, Ambudkar SV. Tyrosine kinase inhibitors as modulators of ABC transporter-mediated drug resistance. Drug Resist Updat 2012;15:70-80.

20. Chen Z, Shi T, Zhang L, Zhu P, Deng M, et al. Mammalian drug efflux transporters of the ATP binding cassette (ABC) family in multidrug resistance: a review of the past decade. Cancer Lett 2016;370:153-64.

21. Liu X. ABC family transporters. In: Pan G, editor. Drug Transporters in Drug Disposition, Effects and Toxicity. Singapore: Springer Singapore; 2019. pp. 13-100.

22. Sharom FJ. The P-glycoprotein multidrug transporter. Essays Biochem 2011;50:161-78.

23. Bloise E, Matthews SG. Chapter 19 - multidrug resistance P-Glycoprotein (P-gp), glucocorticoids, and the stress response. Stress Series: Elsevier; 2019. pp. 227-41.

24. Stouch TR, Gudmundsson O. Progress in understanding the structure-activity relationships of P-glycoprotein. Adv Drug Del Rev 2002;54:315-28.

25. Elmeliegy M, Vourvahis M, Guo C, Wang DD. Effect of P-glycoprotein (P-gp) Inducers on Exposure of P-gp Substrates: review of clinical drug-drug interaction studies. Clin Pharmacokinet 2020; doi: 10.1007/s40262-020-00867-1.

26. Yakusheva EN, Titov DS. Structure and function of multidrug resistance protein 1. Biochemistry (Mosc) 2018;83:907-29.

27. Crawford RR, Potukuchi PK, Schuetz EG, Schuetz JD. Beyond competitive inhibition: regulation of ABC transporters by kinases and protein-protein interactions as potential mechanisms of drug-drug interactions. Drug Metab Dispos 2018;46:567-80.

28. Tolios A, De Las Rivas J, Hovig E, Trouillas P, Scorilas A, et al. Computational approaches in cancer multidrug resistance research: Identification of potential biomarkers, drug targets and drug-target interactions. Drug Resist Updat 2020;48:100662.

29. Dallavalle S, Dobričić V, Lazzarato L, Gazzano E, Machuqueiro M, et al. Improvement of conventional anti-cancer drugs as new tools against multidrug resistant tumors. Drug Resist Updat 2020;50:100682.

30. Cui Q, Wang JQ, Assaraf YG, Ren L, Gupta P, et al. Modulating ROS to overcome multidrug resistance in cancer. Drug Resist Updat 2018;41:1-25.

31. Lee SM, Kim HJ, Kim SY, Kwon MK, Kim S, et al. Drug-loaded gold plasmonic nanoparticles for treatment of multidrug resistance in cancer. Biomaterials 2014;35:2272-82.

32. Calderwood SK. Heat shock proteins and cancer: intracellular chaperones or extracellular signalling ligands? Philos Trans R Soc Lond B Biol Sci 2018;373:20160524.

33. PubChem. Doxorubicin. National Center for Biotechnology Information. USA: NIH; 2020. Available from: https://pubchem.ncbi.nlm.nih. gov/compound/Doxorubicin. [Last accessed on 26 May 2020]

34. Wijdeven RH, Pang B, Assaraf YG, Neefjes J. Old drugs, novel ways out: Drug resistance toward cytotoxic chemotherapeutics. Drug Resist Updat 2016;28:65-81. 
35. Capeloa T, Benyahia Z, Zampieri LX, Blackman M, Sonveaux P. Metabolic and non-metabolic pathways that control cancer resistance to anthracyclines. Semin Cell Dev Biol 2020;98:181-91.

36. PubChem. Cisplatin. National Center for Biotechnology Information. USA: NIH; 2020. Available from: https://pubchem.ncbi.nlm.nih. gov/compound/trans-Dichlorodiamineplatinum_II. [Last accessed on 26 May 2020]

37. Amable L. Cisplatin resistance and opportunities for precision medicine. Pharmacol Res 2016;106:27-36.

38. Morris PG, Fornier MN. Microtubule active agents: beyond the taxane frontier. Clin Cancer Res 2008;14:7167-72.

39. NIH. Docetaxel. LiverTox: Clinical and Research Information on Drug-Induced Liver Injury. Bethesda (MD):National Institute of Diabetes and Digestive and Kidney Diseases; 2012.

40. PubChem. Docetaxel. National Center for Biotechnology Information. USA: NIH; 2020. Available from: https://pubchem.ncbi.nlm.nih. gov/compound/Docetaxel. [Last accessed on 26 May 2020]

41. Farha NG, Kasi A. Docetaxel. StatPearls. Treasure Island (FL): StatPearls Publishing LLC.; 2020.

42. Lee S, Kim K, Ho JN, Jin H, Byun SS, et al. Analysis of resistance-associated gene expression in docetaxel-resistant prostate cancer cells. Oncol Lett 2017;14:3011-8.

43. Shen DW, Pouliot LM, Hall MD, Gottesman MM. Cisplatin resistance: a cellular self-defense mechanism resulting from multiple epigenetic and genetic changes. Pharmacol Rev 2012;64:706-21.

44. Manohar S, Leung N. Cisplatin nephrotoxicity: a review of the literature. J Nephrol 2018;31:15-25.

45. Ciarimboli G. Membrane transporters as mediators of cisplatin side-effects. Anticancer Res 2014;34:547-50.

46. NIH. Precision medicine in cancer treatment. Available from: https://www.cancer.gov/about-cancer/treatment/types/precision-medicine. [Last accessed on 26 May 2020]

47. Chatterjee N, Bivona TG. Polytherapy and targeted cancer drug resistance. Trends Cancer 2019;5:170-82.

48. Sabnis AJ, Bivona TG. Principles of resistance to targeted cancer therapy: lessons from basic and translational cancer biology. Trends Mol Med 2019;25:185-97.

49. Sarmento-Ribeiro AB, Scorilas A, Gonçalves AC, Efferth T, Trougakos IP. The emergence of drug resistance to targeted cancer therapies: clinical evidence. Drug Resistance Updates 2019;47:100646.

50. Whirl-Carrillo M, McDonagh EM, Hebert JM, Gong L, Sangkuhl K, et al. Pharmacogenomics knowledge for personalized medicine. Clin Pharmacol Ther 2012;92:414-7.

51. Shi J, Kantoff PW, Wooster R, Farokhzad OC. Cancer nanomedicine: progress, challenges and opportunities. Nat Rev Cancer 2017;17:2037.

52. Su H, Wang Y, Gu Y, Bowman L, Zhao J, et al. Potential applications and human biosafety of nanomaterials used in nanomedicine. J Appl Toxicol 2018;38:3-24.

53. Stepensky D. Prediction of drug disposition on the basis of its chemical structure. Clin Pharmacokinet 2013;52:415-31.

54. Gulati NM, Stewart PL, Steinmetz NF. Bioinspired shielding strategies for nanoparticle drug delivery applications. Mol Pharm 2018;15:2900-9.

55. Rejman J, Oberle V, Zuhorn IS, Hoekstra D. Size-dependent internalization of particles via the pathways of clathrin- and caveolaemediated endocytosis. Biochem J 2004;377:159-69.

56. Morales-Cruz M, Delgado Y, Castillo B, Figueroa CM, Molina AM, et al. Smart Targeting to improve cancer therapeutics. Drug Des Devel Ther 2019;13:3753-72.

57. Bjornmalm M, Thurecht KJ, Michael M, Scott AM, Caruso F. Bridging Bio-Nano Science and Cancer Nanomedicine. ACS Nano 2017;11:9594-613.

58. Thomas OS, Weber W. Overcoming physiological barriers to nanoparticle delivery - are we there yet? Frontiers in Bioengineering and Biotechnology 2019;7:415.

59. Sindhwani S, Syed AM, Ngai J, Kingston BR, Maiorino L, et al. The entry of nanoparticles into solid tumours. Nat Mater 2020; doi: 10.1038/s41563-019-0566-2.

60. Bazak R, Houri M, El Achy S, Kamel S, Refaat T. Cancer active targeting by nanoparticles: a comprehensive review of literature. J Cancer Res Clin Oncol 2015;141:769-84.

61. Roma-Rodrigues C, Pombo I, Raposo L, Pedrosa P, Fernandes AR, et al. Nanotheranostics targeting the tumor microenvironment. Front Bioeng Biotechnol 2019;7:197.

62. ClinicalTrials.gov. A Study of CriPec $®$ Docetaxel Given to Patients With Solid Tumours (NAPOLY). Available from: https://clinicaltrials. gov/ct2/show/NCT02442531. [Last accessed on 26 May 2020]

63. Atrafi F, van Eerden RAG, van Hylckama Vlieg MAM, Oomen-de Hoop E, de Bruijn P, et al. Intratumoral comparison of nanoparticle entrapped docetaxel (CPC634) with conventional docetaxel in patients with solid tumors. Clin Cancer Res 2020; doi: 10.1158/1078-0432. CCR-20-0008.

64. Zhang M, Chen X, Li C, Shen X. Charge-reversal nanocarriers: an emerging paradigm for smart cancer nanomedicine. J Control Release 2019;319:46-62.

65. Xu J, Gulzar A, Yang D, Gai S, He F, et al. Tumor self-responsive upconversion nanomedicines for theranostic applications. Nanoscale 2019;11:17535-56.

66. De Santis MC, Porporato PE, Martini M, Morandi A. Signaling pathways regulating redox balance in cancer metabolism. Front Oncol 2018;8:126.

67. Llopis J, McCaffery JM, Miyawaki A, Farquhar MG, Tsien RY. Measurement of cytosolic, mitochondrial, and Golgi pH in single living cells with green fluorescent proteins. Proc Natl Acad Sci U S A 1998;95:6803-8. 
68. Benham AM. Endoplasmic reticulum redox pathways: in sickness and in health. FEBS J 2019;286:311-21.

69. Jose J, Kumar R, Harilal S, Mathew GE, Parambi DGT, et al. Magnetic nanoparticles for hyperthermia in cancer treatment: an emerging tool. Environ Sci Pollut Res Int 2019; doi: 10.1007/s11356-019-07231-2.

70. Chandrasekharan P, Tay ZW, Hensley D, Zhou XY, Fung BK, et al. Using magnetic particle imaging systems to localize and guide magnetic hyperthermia treatment: tracers, hardware, and future medical applications. Theranostics 2020;10:2965-81.

71. Wang H, Agarwal P, Liang Y, Xu J, Zhao G, et al. Enhanced cancer therapy with cold-controlled drug release and photothermal warming enabled by one nanoplatform. Biomaterials 2018;180:265-78.

72. Hou Y, Sun Z, Rao W, Liu J. Nanoparticle-mediated cryosurgery for tumor therapy. Nanomedicine 2018;14:493-506.

73. Papa AL, Korin N, Kanapathipillai M, Mammoto A, Mammoto T, et al. Ultrasound-sensitive nanoparticle aggregates for targeted drug delivery. Biomaterials 2017;139:187-94.

74. Karimi M, Ghasemi A, Sahandi Zangabad P, Rahighi R, et al. Smart micro/nanoparticles in stimulus-responsive drug/gene delivery systems. Chem Soc Rev 2016;45:1457-501.

75. Ai X, Mu J, Xing B. Recent advances of light-mediated theranostics. Theranostics 2016;6:2439-57.

76. Kobayashi H, Ogawa M, Alford R, Choyke PL, Urano Y. New strategies for fluorescent probe design in medical diagnostic imaging. Chem Rev 2009;110:2620-40.

77. Sinha RP, Hader DP. UV-induced DNA damage and repair: a review. Photochem Photobiol Sci 2002;1:225-36.

78. Kochevar IE. UV-induced protein alterations and lipid oxidation in erythrocyte membranes. Photochem photobiol 1990;52:795-800.

79. Ash C, Dubec M, Donne K, Bashford T. Effect of wavelength and beam width on penetration in light-tissue interaction using computational methods. Lasers Med Sci 2017;32:1909-18.

80. He S, Song J, Qu J, Cheng Z. Crucial breakthrough of second near-infrared biological window fluorophores: design and synthesis toward multimodal imaging and theranostics. Chem Soc Rev 2018;47:4258-78.

81. Zhao J, Zhong D, Zhou S. NIR-I-to-NIR-II fluorescent nanomaterials for biomedical imaging and cancer therapy. J Mater Chem B 2018;6:349-65.

82. Henderson TA, Morries LD. Near-infrared photonic energy penetration: can infrared phototherapy effectively reach the human brain? Neuropsychiatr Dis Treat 2015;11:2191-208.

83. Liu B, Li C, Cheng Z, Hou Z, Huang S, Lin J. Functional nanomaterials for near-infrared-triggered cancer therapy. Biomater Sci 2016;4:890-909.

84. Khan I, Tang E, Arany P. Molecular pathway of near-infrared laser phototoxicity involves ATF-4 orchestrated ER stress. Sci Rep 2015;5:10581.

85. Chu KF, Dupuy DE. Thermal ablation of tumours: biological mechanisms and advances in therapy. Nat Rev Cancer 2014;14:199-208.

86. Nikfarjam M, Muralidharan V, Christophi C. Mechanisms of focal heat destruction of liver tumors. J Surg Res 2005;127:208-23.

87. Doughty ACV, Hoover AR, Layton E, Murray CK, Howard EW, et al. Nanomaterial applications in photothermal therapy for cancer. Materials (Basel, Switzerland) 2019;12:779.

88. Wang L, Lin X, Wang J, Hu Z, Ji Y, et al. Novel insights into combating cancer chemotherapy resistance using a plasmonic nanocarrier: enhancing drug sensitiveness and accumulation simultaneously with localized mild photothermal stimulus of femtosecond pulsed laser. Adv Funct Mater 2014;24:4229-39.

89. Ashikbayeva Z, Tosi D, Balmassov D, Schena E, Saccomandi P, et al. Application of nanoparticles and nanomaterials in thermal ablation therapy of cancer. Nanomaterials (Basel) 2019;9:E1195.

90. Svaasand LO, Gomer CJ, Morinelli E. On the physical rationale of laser induced hyperthermia. Lasers Med Sci 1990;5:121-8.

91. Jung HS, Verwilst P, Sharma A, Shin J, Sessler JL, et al. Organic molecule-based photothermal agents: an expanding photothermal therapy universe. Chem Soc Rev 2018;47:2280-97.

92. Samadi A, Klingberg H, Jauffred L, Kjaer A, Bendix PM, et al. Platinum nanoparticles: a non-toxic, effective and thermally stable alternative plasmonic material for cancer therapy and bioengineering. Nanoscale 2018;10:9097-107.

93. Dolmans DE, Fukumura D, Jain RK. Photodynamic therapy for cancer. Nat Rev Cancer 2003;3:380-7.

94. Agostinis P, Berg K, Cengel KA, Foster TH, Girotti AW, et al. Photodynamic therapy of cancer: an update. CA Cancer J Clin 2011;61:250-81.

95. Sorrin AJ, Kemal Ruhi M, Ferlic NA, Karimnia V, Polacheck WJ, et al. Photodynamic therapy and the biophysics of the tumor microenvironment. Photochem Photobiol 2020;96:232-59.

96. Spring BQ, Rizvi I, Xu N, Hasan T. The role of photodynamic therapy in overcoming cancer drug resistance. Photochem Photobiol Sci 2015;14:1476-91.

97. Russell LM, Liu CH, Grodzinski P. Nanomaterials innovation as an enabler for effective cancer interventions. Biomaterials 2020;242:119926.

98. Aldieri E, Bergandi L, Riganti C, Costamagna C, Bosia A, et al. Doxorubicin induces an increase of nitric oxide synthesis in rat cardiac cells that is inhibited by iron supplementation. Toxicol Appl Pharmacol 2002;185:85-90.

99. Riganti C, Miraglia E, Viarisio D, Costamagna C, Pescarmona G, et al. Nitric oxide reverts the resistance to doxorubicin in human colon cancer cells by inhibiting the drug efflux. Cancer Res 2005;65:516-25.

100. Delou JMA, Souza ASO, Souza LCM, Borges HL. Highlights in resistance mechanism pathways for combination therapy. Cells 2019;8:1013.

101. Hu CMJ, Aryal S, Zhang L. Nanoparticle-assisted combination therapies for effective cancer treatment. Ther Deliv 2010;1:323-34.

102. Jiang Y, Guo Z, Fang J, Wang B, Lin Z, et al. A multi-functionalized nanocomposite constructed by gold nanorod core with triple-layer 
coating to combat multidrug resistant colorectal cancer. Mater Sci Eng C Mater Biol Appl 2020;107:110224.

103. Dong J, Qin Z, Zhang WD, Cheng G, Yehuda AG, et al. Medicinal chemistry strategies to discover P-glycoprotein inhibitors: an update. Drug Resist Updat 2020;49:100681.

104. Hu CM, Zhang L. Nanoparticle-based combination therapy toward overcoming drug resistance in cancer. Biochem Pharmacol 2012;83:1104-11.

105. Zhang M, Liu E, Cui Y, Huang Y. Nanotechnology-based combination therapy for overcoming multidrug-resistant cancer. Cancer Biol Med 2017;14:212-27.

106. Luo D, Carter KA, Miranda D, Lovell JF. Chemophototherapy: an emerging treatment option for solid tumors. Adv Sci (Weinh) 2017;4:1600106.

107. Sagar V, Nair M. Near-infrared biophotonics-based nanodrug release systems and their potential application for neuro-disorders. Expert Opin Drug Deliv 2018;15:137-52.

108. Goodman AM, Neumann O, Nørregaard K, Henderson L, Choi MR, et al. Near-infrared remotely triggered drug-release strategies for cancer treatment. Proc Natl Acad Sci U S A 2017;114:12419-24.

109. Ke W, Yu P, Wang J, Wang R, Guo C, et al. MCF-7/ADR cells (re-designated NCI/ADR-RES) are not derived from MCF-7 breast cancer cells: a loss for breast cancer multidrug-resistant research. Med Oncol 2011;28 Suppl 1:S135-41.

110. Leroy B, Girard L, Hollestelle A, Minna JD, Gazdar AF, et al. Analysis of TP53 mutation status in human cancer cell lines: a reassessment. Hum Mutat 2014;35:756-65.

111. Chen S, Lei Q, Qiu WX, Liu LH, Zheng DW, et al. Mitochondria-targeting "Nanoheater" for enhanced photothermal/chemo-therapy. Biomaterials 2017;117:92-104.

112. Zhang Z, Xu S, Wang Y, Yu Y, Li F, et al. Near-infrared triggered co-delivery of doxorubicin and quercetin by using gold nanocages with tetradecanol to maximize anti-tumor effects on MCF-7/ADR cells. J Colloid Interface Sci 2018;509:47-57.

113. Kang S, Kang K, Chae A, Kim YK, Jang H, et al. Fucoidan-coated coral-like Pt nanoparticles for computed tomography-guided highly enhanced synergistic anticancer effect against drug-resistant breast cancer cells. Nanoscale 2019;11:15173-83.

114. Zhang Y, Sha R, Zhang L, Zhang W, Jin P, et al. Harnessing copper-palladium alloy tetrapod nanoparticle-induced pro-survival autophagy for optimized photothermal therapy of drug-resistant cancer. Nat Commun 2018;9:4236.

115. Zeng L, Pan Y, Tian Y, Wang X, Ren W, et al. Doxorubicin-loaded NaYF4:Yb/Tm-TiO2 inorganic photosensitizers for NIR-triggered photodynamic therapy and enhanced chemotherapy in drug-resistant breast cancers. Biomaterials 2015;57:93-106.

116. Jiao X, Wang Z, Wang F, Wen Y. Dual stimuli-responsive controlled release nanocarrier for multidrug resistance cancer therapy. Chemphyschem 2019;20:3271-5.

117. Dong X, Yin W, Zhang X, Zhu S, He X, et al. Intelligent MoS2 nanotheranostic for targeted and Enzyme-/pH-/NIR-responsive drug delivery to overcome cancer chemotherapy resistance guided by PET imaging. ACS Appl Mater Interfaces 2018;10:4271-84.

118. Guo R, Tian Y, Wang Y, Yang W. Near-infrared laser-triggered nitric oxide nanogenerators for the reversal of multidrug resistance in cancer. Adv Funct Mater 2017;27:1606398.

119. Feng L, Li K, Shi X, Gao M, Liu J, et al. Smart pH-responsive nanocarriers based on nano-graphene oxide for combined chemo- and photothermal therapy overcoming drug resistance. Adv Healthc Mater 2014;3:1261-71.

120. Zeng X, Luo M, Liu G, Wang X, Tao W, et al. Polydopamine-modified black phosphorous nanocapsule with enhanced stability and photothermal performance for tumor multimodal treatments. Adv Sci (Weinh) 2018;5:1800510.

121. Wu F, Zhang M, Chu X, Zhang Q, Su Y, et al. Black phosphorus nanosheets-based nanocarriers for enhancing chemotherapy drug sensitiveness via depleting mutant p53 and resistant cancer multimodal therapy. Chem Eng J 2019;370:387-99.

122. Li H, Liu C, Zeng YP, Hao YH, Huang JW, et al. Nanoceria-mediated drug delivery for targeted photodynamic therapy on drug-resistant breast cancer. ACS Appl Mater Interfaces 2016;8:31510-23.

123. Yao C, Wang P, Li X, Hu X, Hou J, et al. Near-infrared-triggered azobenzene-liposome/upconversion nanoparticle hybrid vesicles for remotely controlled drug delivery to overcome cancer multidrug resistance. Adv Mater 2016;28:9341-8.

124. Wang L, Sun Q, Wang X, Wen T, Yin JJ, et al. Using hollow carbon nanospheres as a light-induced free radical generator to overcome chemotherapy resistance. J Am Chem Soc 2015;137:1947-55.

125. Pai CL, Chen YC, Hsu CY, Su HL, Lai PS. Carbon nanotube-mediated photothermal disruption of endosomes/lysosomes reverses doxorubicin resistance in MCF-7/ADR cells. J Biomed Nanotechnol 2016;12:619-29.

126. Kalluru P, Vankayala R, Chiang CS, Hwang KC. Unprecedented "All-in-One" lanthanide-doped mesoporous silica frameworks for fluorescence/MR imaging and combination of nir light triggered chemo-photodynamic therapy of tumors. Adv Funct Mater 2016;26:7908-20.

127. Ding Y, Du C, Qian J, Dong CM. NIR-responsive polypeptide nanocomposite generates NO gas, mild photothermia, and chemotherapy to reverse multidrug-resistant cancer. Nano Lett 2019;19:4362-70.

128. Wei G, Yang G, Wei B, Wang Y, Zhou S. Near-infrared light switching nitric oxide nanoemitter for triple-combination therapy of multidrug resistant cancer. Acta Biomater 2019;100:365-77.

129. Peng Y, Nie J, Cheng W, Liu G, Zhu D, et al. A multifunctional nanoplatform for cancer chemo-photothermal synergistic therapy and overcoming multidrug resistance. Biomater Sci 2018;6:1084-98.

130. Lai GM, Chen YN, Mickley LA, Fojo AT, Bates SE. P-glycoprotein expression and schedule dependence of adriamycin cytotoxicity in human colon carcinoma cell lines. Int J Cancer 1991;49:696-703.

131. Ai F, Sun T, Xu Z, Wang Z, Kong W, et al. An upconversion nanoplatform for simultaneous photodynamic therapy and Pt chemotherapy to combat cisplatin resistance. Dalton Trans 2016;45:13052-60. 
132. Zhang J, Ma Y, Hu K, Feng Y, Chen S, et al. Surface coordination of black phosphorus with modified cisplatin. Bioconjug Chem 2019;30:1658-64.

133. Tran TH, Nguyen HT, Pham TT, Choi JY, Choi HG, et al. Development of a graphene oxide nanocarrier for dual-drug chemophototherapy to overcome drug resistance in cancer. ACS Appl Mater Interfaces 2015;7:28647-55.

134. Balaji SA, Udupa N, Chamallamudi MR, Gupta V, Rangarajan A. Role of the drug transporter ABCC3 in breast cancer chemoresistance. PLoS One 2016;11:e155013.

135. Roll JD, Rivenbark AG, Jones WD, Coleman WB. DNMT3b overexpression contributes to a hypermethylator phenotype in human breast cancer cell lines. Mol Cancer 2008;7:1-15.

136. Poulose AC, Veeranarayanan S, Mohamed MS, Aburto RR, Mitcham T, et al. Multifunctional Cu2-xTe nanocubes mediated combination therapy for multi-drug resistant MDA MB 453. Sci Rep 2016;6:35961.

137. Singh G, Odriozola L, Guan H, Kennedy CR, Chan AM. Characterization of a novel PTEN mutation in MDA-MB-453 breast carcinoma cell line. BMC Cancer 2011;11:490.

138. Li R, Wu R, Zhao L, Wu M, Yang L, et al. P-glycoprotein antibody functionalized carbon nanotube overcomes the multidrug resistance of human leukemia cells. ACS Nano 2010;4:1399-408.

139. Hu M, Liu Y, Deng C, Han R, Jia Y, et al. Enhanced invasiveness in multidrug resistant leukemic cells is associated with overexpression of P-glycoprotein and cellular inhibitor of apoptosis protein. Leuk Lymphoma 2011;52:1302-11.

140. Mattheolabakis G, Milane L, Singh A, Amiji MM. Hyaluronic acid targeting of CD44 for cancer therapy: from receptor biology to nanomedicine. J Drug Target 2015;23:605-18.

141. Zhong Y, Zhang J, Cheng R, Deng C, Meng F, et al. Reversibly crosslinked hyaluronic acid nanoparticles for active targeting and intelligent delivery of doxorubicin to drug resistant CD44+ human breast tumor xenografts. J Control Release 2015;205:144-54.

142. Chanmee T, Ontong P, Itano N. Hyaluronan: a modulator of the tumor microenvironment. Cancer Lett 2016;375:20-30.

143. PubChem. Folic acid. National Center for Biotechnology Information. USA: NIH; 2020. Available from: https://pubchem.ncbi.nlm.nih. gov/compound/Folic-acid. [Last accessed on 26 May 2020]

144. Jahangirian H, Kalantari K, Izadiyan Z, Rafiee-Moghaddam R, Shameli K, et al. A review of small molecules and drug delivery applications using gold and iron nanoparticles. Int J Nanomedicine 2019;14:1633-57.

145. Choudhury H, Pandey M, Chin PX, Phang YL, Cheah JY, et al. Transferrin receptors-targeting nanocarriers for efficient targeted delivery and transcytosis of drugs into the brain tumors: a review of recent advancements and emerging trends. Drug Deliv Transl Res 2018;8:1545-63.

146. PubChem. Quercetin. National Center for Biotechnology Information. USA: NIH; 2020. Available from: https://pubchem.ncbi.nlm.nih. gov/compound/Quercetin. [Last accessed on 26 May 2020]

147. PubChem. 3-Methyladenine. National Center for Biotechnology Information. USA: NIH; 2020. Available from: https://pubchem.ncbi. nlm.nih.gov/compound/3-Methyladenine. [Last accessed on 26 May 2020]

148. PubChem. Chloroquine. National Center for Biotechnology Information. USA: NIH; 2020. Available from: https://pubchem.ncbi.nlm.nih. gov/compound/Chloroquine. [Last accessed on 26 May 2020]

149. PubChem. Tocophersolan. National Center for Biotechnology Information. USA: NIH; 2020. Available from: https://pubchem.ncbi.nlm. nih.gov/compound/Tocophersolan. [Last accessed on 26 May 2020]

150. PubChem. 1-Tetradecanol. National Center for Biotechnology Information, USA: NIH; 2020. Available from: https://pubchem.ncbi.nlm. nih.gov/compound/1-Tetradecanol. [Last accessed on 26 May 2020]

151. PubChem. Irinotecan. National Center for Biotechnology Information, USA: NIH; 2020. Available from: https://pubchem.ncbi.nlm.nih. gov/compound/Irinotecan. [Last accessed on 26 May 2020]

152. Reyhanoglu G, Smith T. Irinotecan. StatPearls. Treasure Island (FL): StatPearls Publishing LLC.; 2020.

153. Huang J, Si L, Jiang L, Fan Z, Qiu J, et al. Effect of pluronic F68 block copolymer on P-glycoprotein transport and CYP3A4 metabolism. Int J Pharm 2008;356:351-3.

154. PubChem. Poloxamer 188. National Center for Biotechnology Information. USA: NIH; 2020. Available from: https://pubchem.ncbi.nlm. nih.gov/compound/Poloxamer-188. [Last accessed on 26 May 2020]

155. Suk JS, Xu Q, Kim N, Hanes J, Ensign LM. PEGylation as a strategy for improving nanoparticle-based drug and gene delivery. Adv Drug Deliv Rev 2016;99:28-51.

156. Willers C, Svitina H, Rossouw MJ, Swanepoel RA, Hamman JH, et al. Models used to screen for the treatment of multidrug resistant cancer facilitated by transporter-based efflux. J Cancer Res Clin Oncol 2019;145:1949-76.

157. Li J, Zhang W, Gao Y, Tong H, Chen Z, et al. Near-infrared light and magnetic field dual-responsive porous silicon-based nanocarriers to overcome multidrug resistance in breast cancer cells with enhanced efficiency. J Mater Chem B 2020;8:546-57.

158. Langhans SA. Three-dimensional in vitro cell culture models in drug discovery and drug repositioning. Front Pharmacol 2018;9:6.

159. Costa EC, Moreira AF, de Melo-Diogo D, Gaspar VM, Carvalho MP, et al. 3D tumor spheroids: an overview on the tools and techniques used for their analysis. Biotechnol Adv 2016;34:1427-41.

160. Nunes AS, Barros AS, Costa EC, Moreira AF, Correia IJ. 3D tumor spheroids as in vitro models to mimic in vivo human solid tumors resistance to therapeutic drugs. Biotechnol Bioeng 2019;116:206-26.

161. Lu H, Stenzel MH. Multicellular tumor spheroids (MCTS) as a 3D in vitro evaluation tool of nanoparticles. Small 2018;14:e1702858.

162. Walker JV, Nitiss JL. DNA topoisomerase II as a target for cancer chemotherapy. Cancer Invest 2002;20:570-89.

163. Tang S, Chen M, Zheng N. Sub-10-nm Pd nanosheets with renal clearance for efficient near-infrared photothermal cancer therapy. Small 2014;10:3139-44 
164. Xiao JW, Fan SX, Wang F, Sun LD, Zheng XY, et al. Porous Pd nanoparticles with high photothermal conversion efficiency for efficient ablation of cancer cells. Nanoscale 2014;6:4345-51.

165. Jalani G, Tam V, Vetrone F, Cerruti M. Seeing, targeting and delivering with upconverting nanoparticles. J Am Chem Soc 2018;140:10923-31.

166. Hessel CM, Pattani VP, Rasch M, Panthani MG, Koo B, et al. Copper selenide nanocrystals for photothermal therapy. Nano Lett 2011;11:2560-6.

167. Li W, Zamani R, Rivera Gil P, Pelaz B, Ibanez M, et al. CuTe nanocrystals: shape and size control, plasmonic properties, and use as SERS probes and photothermal agents. J Am Chem Soc 2013;135:7098-101.

168. Wang S, Riedinger A, Li H, Fu C, Liu H, et al. Plasmonic copper sulfide nanocrystals exhibiting near-infrared photothermal and photodynamic therapeutic effects. ACS Nano 2015;9:1788-800.

169. Chen X, Yang J, Wu T, Li L, Luo W, et al. Nanostructured binary copper chalcogenides: synthesis strategies and common applications. Nanoscale 2018;10:15130-63.

170. Tian Q, Tang M, Sun Y, Zou R, Chen Z, et al. Hydrophilic flower-like CuS superstructures as an efficient $980 \mathrm{~nm}$ laser-driven photothermal agent for ablation of cancer cells. Adv Mater 2011;23:3542-7.

171. Wu F, Zhang M, Lu H, Liang D, Huang Y, et al. Triple stimuli-responsive magnetic hollow porous carbon-based nanodrug delivery system for magnetic resonance imaging-guided synergistic photothermal/chemotherapy of cancer. ACS Appl Mater Interfaces 2018;10:21939-49.

172. Jain PK, Huang X, El-Sayed IH, El-Sayed MA. Review of some interesting surface plasmon resonance-enhanced properties of noble metal nanoparticles and their applications to biosystems. Plasmonics 2007;2:107-18.

173. Vines JB, Yoon JH, Ryu NE, Lim DJ, Park H. Gold nanoparticles for photothermal cancer therapy. Front Chem 2019;7:167.

174. Agrawal A, Johns RW, Milliron DJ. Control of localized surface plasmon resonances in metal oxide nanocrystals. Annu Rev Mater Res 2017;47:1-31.

175. Kohout C, Santi C, Polito L. Anisotropic gold nanoparticles in biomedical applications. Int J Mol Sci 2018;19:3385.

176. Kodiha M, Wang YM, Hutter E, Maysinger D, Stochaj U. Off to the organelles - killing cancer cells with targeted gold nanoparticles. Theranostics 2015;5:357-70.

177. Venditti I. Engineered gold-based nanomaterials: morphologies and functionalities in biomedical applications. Bioengineering (Basel) 2019;6:53.

178. Chauhan VP, Popović Z, Chen O, Cui J, Fukumura D, et al. Fluorescent nanorods and nanospheres for real-time in vivo probing of nanoparticle shape-dependent tumor penetration. Angew Chem Int Ed Engl 2011;50:11417-20.

179. Samhadaneh DM, Chu S, Maysinger D, Stochaj U. How could gold nanourchins be applied in the clinic? Nanomedicine 2019;15:829-32.

180. Kodiha M, Hutter E, Boridy S, Juhas M, Maysinger D, et al. Gold nanoparticles induce nuclear damage in breast cancer cells which is further amplified by hyperthermia. CMLS 2014;71:4259-73.

181. Karimi M, Zangabad PS, Mehdizadeh F, Malekzad H, Ghasemi A, et al. Nanocaged platforms: modification, drug delivery and nanotoxicity. Opening synthetic cages to release the tiger. Nanoscale 2017;9:1356-92.

182. Knowlton AA. NFkappaB, heat shock proteins, HSF-1, and inflammation. Cardiovasc Res 2006;69:7-8.

183. Atashrazm F, Lowenthal RM, Woods GM, Holloway AF, Dickinson JL. Fucoidan and cancer: a multifunctional molecule with anti-tumor potential. Mar Drugs 2015;13:2327-46.

184. Pons-Tostivint E, Thibault B, Guillermet-Guibert J. Targeting PI3K Signaling in Combination Cancer Therapy. Trends Cancer 2017;3:454-69.

185. Bhat P, Kriel J, Shubha Priya B, Basappa, Shivananju NS, Loos B. Modulating autophagy in cancer therapy: advancements and challenges for cancer cell death sensitization. Biochem Pharmacol 2018;147:170-82.

186. Tessitore G, Maurizio SL, Sabri T, Capobianco JA. Intrinsic time-tunable emissions in core-shell upconverting nanoparticle systems. Angew Chem Int Ed Engl 2019;58:9742-51.

187. Oliveira H, Bednarkiewicz A, Falk A, Fröhlich E, Lisjak D, et al. Critical considerations on the clinical translation of upconversion nanoparticles (UCNPs): recommendations from the European Upconversion Network (COST Action CM1403). Adv Healthc Mater 2019;8:e1801233.

188. Hu XY, Jia K, Cao Y, Li Y, Qin S, et al. Dual photo- and pH-responsive supramolecular nanocarriers based on water-soluble pillar[6]arene and different azobenzene derivatives for intracellular anticancer drug delivery. Chemistry (Easton) 2015;21:1208-20.

189. Comin A, Manna L. New materials for tunable plasmonic colloidal nanocrystals. Chem Soc Rev 2014;43:3957-75.

190. Liu K, Liu K, Liu J, Ren Q, Zhao Z, et al. Copper chalcogenide materials as photothermal agents for cancer treatment. Nanoscale 2020;12:2902-13.

191. Tan C, Zhang H. Two-dimensional transition metal dichalcogenide nanosheet-based composites. Chem Soc Rev 2015;44:2713-31.

192. Agarwal V, Chatterjee K. Recent advances in the field of transition metal dichalcogenides for biomedical applications. Nanoscale 2018;10:16365-97.

193. Chhowalla M, Shin HS, Eda G, Li LJ, Loh KP, et al. The chemistry of two-dimensional layered transition metal dichalcogenide nanosheets. Nat Chem 2013;5:263-75.

194. Liu T, Wang C, Gu X, Gong H, Cheng L, et al. Drug delivery with PEGylated $\mathrm{MoS}_{2}$ nano-sheets for combined photothermal and chemotherapy of cancer. Adv Mater 2014;26:3433-40.

195. Wang C, Bai J, Liu Y, Jia X, Jiang X. Polydopamine coated selenide molybdenum: a new photothermal nanocarrier for highly effective chemo-photothermal synergistic therapy. ACS Biomater Sci Engineering 2016;2:2011-7.

196. Wang Y, Zhang F, Wang Q, Yang P, Lin H, et al. Hierarchical MoSe2 nanoflowers as novel nanocarriers for NIR-light-mediated 
synergistic photo-thermal/dynamic and chemo-therapy. Nanoscale 2018;10:14534-45.

197. Wang Y, Zhao J, Chen Z, Zhang F, Wang Q, et al. Construct of MoSe2/Bi2Se3 nanoheterostructure: Multimodal CT/PT imaging-guided PTT/PDT/chemotherapy for cancer treating. Biomaterials 2019;217:119282.

198. Liao W, Zhang L, Zhong Y, Shen Y, Li C, et al. Fabrication of ultrasmall WS2 quantum dots-coated periodic mesoporous organosilica nanoparticles for intracellular drug delivery and synergistic chemo-photothermal therapy. Onco Targets Ther 2018;11:1949-60.

199. Tammi MI, Oikari S, Pasonen-Seppanen S, Rilla K, Auvinen P, et al. Activated hyaluronan metabolism in the tumor matrix - Causes and consequences. Matrix Biol 2019;78-79:147-64.

200. Muzzio M, Li J, Yin Z, Delahunty IM, Xie J, et al. Monodisperse nanoparticles for catalysis and nanomedicine. Nanoscale 2019;11:18946-67.

201. Shah A, Dobrovolskaia MA. Immunological effects of iron oxide nanoparticles and iron-based complex drug formulations: therapeutic benefits, toxicity, mechanistic insights, and translational considerations. Nanomedicine 2018;14:977-90.

202. Li J, Wang S, Shi X, Shen M. Aqueous-phase synthesis of iron oxide nanoparticles and composites for cancer diagnosis and therapy. Adv Colloid Interface Sci 2017;249:374-85.

203. Wang M, Hou Z, Al Kheraif AA, Xing B, Lin J. Mini Review of $\mathrm{TiO}_{2}$-based multifunctional nanocomposites for near-infrared lightresponsive phototherapy. Adv Healthc Mater 2018;7:e1800351.

204. Raja G, Cao S, Kim DH, Kim TJ. Mechanoregulation of titanium dioxide nanoparticles in cancer therapy. Mater Sci Eng C Mater Biol Appl 2020;107:110303.

205. Ziental D, Czarczynska-Goslinska B, Mlynarczyk DT, Glowacka-Sobotta A, Stanisz B, et al. Titanium dioxide nanoparticles: prospects and applications in medicine. Nanomaterials (Basel) 2020;10:387.

206. Popov AL, Han B, Ermakov AM, Savintseva IV, Ermakova ON, et al. PVP-stabilized tungsten oxide nanoparticles: pH sensitive anticancer platform with high cytotoxicity. Mater Sci Eng C Mater Biol Appl 2020;108:110494.

207. Huo D, Zhu J, Chen G, Chen Q, Zhang C, et al. Eradication of unresectable liver metastasis through induction of tumour specific energy depletion. Nat Commun 2019;10:3051.

208. Tian Y, Yi W, Bai L, Zhang P, Si J, et al. Lentinan in-situ coated tungsten oxide nanorods as a nanotherapeutic agent for low power density photothermal cancer therapy. Int J Biol Macromol 2019;137:904-11.

209. Chang D, Lim M, Goos J, Qiao R, Ng YY, et al. Biologically targeted magnetic hyperthermia: potential and limitations. Front Pharmacol 2018;9:831

210. Mohapatra J, Xing M, Liu JP. Inductive thermal effect of ferrite magnetic nanoparticles. Materials (Basel) 2019;12:3208.

211. Estelrich J, Escribano E, Queralt J, Busquets MA. Iron oxide nanoparticles for magnetically-guided and magnetically-responsive drug delivery. Int J Mol Sci 2015;16:8070-101.

212. Loh KP, Ho D, Chiu GNC, Leong DT, Pastorin G, et al. Clinical applications of carbon nanomaterials in diagnostics and therapy. Adv Mater 2018;30:e1802368.

213. Augustine S, Singh J, Srivastava M, Sharma M, Das A, et al. Recent advances in carbon based nanosystems for cancer theranostics. Biomater Sci 2017;5:901-52.

214. Yuan X, Zhang X, Sun L, Wei Y, Wei X. Cellular toxicity and immunological effects of carbon-based nanomaterials. Part Fibre Toxicol $2019 ; 16: 18$

215. Yang K, Feng L, Liu Z. Stimuli responsive drug delivery systems based on nano-graphene for cancer therapy. Adv Drug Deliv Rev 2016;105:228-41.

216. Vankayala R, Hwang KC. Near-infrared-light-activatable nanomaterial-mediated phototheranostic nanomedicines: an emerging paradigm for cancer treatment. Adv Mater 2018;30:e1706320.

217. Patel KD, Singh RK, Kim HW. Carbon-based nanomaterials as an emerging platform for theranostics. Mater Horiz 2019;6:434-69.

218. de Melo-Diogo D, Lima-Sousa R, Alves CG, Correia IJ. Graphene family nanomaterials for application in cancer combination photothermal therapy. Biomater Sci 2019;7:3534-51.

219. Hamblin MR. Fullerenes as photosensitizers in photodynamic therapy: pros and cons. Photochem Photobiol Sci 2018;17:1515-33.

220. Wong BS, Yoong SL, Jagusiak A, Panczyk T, Ho HK, et al. Carbon nanotubes for delivery of small molecule drugs. Adv Drug Deliv Rev 2013;65:1964-2015.

221. Cha C, Shin SR, Annabi N, Dokmeci MR, Khademhosseini A. Carbon-based nanomaterials: multifunctional materials for biomedical engineering. ACS Nano 2013;7:2891-7.

222. Negri V, Pacheco-Torres J, Calle D, López-Larrubia P. Carbon Nanotubes in biomedicine. Top Curr Chem (Cham) 2020;378:15.

223. Kam NWS, O’Connell M, Wisdom JA, Dai H. Carbon nanotubes as multifunctional biological transporters and near-infrared agents for selective cancer cell destruction. Proc Natl Acad Sci U S A 2005;102:11600-5.

224. Tran HQ, Batul R, Bhave M, Yu A. Current advances in the utilization of polydopamine nanostructures in biomedical therapy. Biotechnol J 2019;14:e1900080.

225. Wang Z, Duan Y, Duan Y. Application of polydopamine in tumor targeted drug delivery system and its drug release behavior. J Control Release 2018;290:56-74.

226. Choi JR, Yong KW, Choi JY, Nilghaz A, Lin Y, et al. Black phosphorus and its biomedical applications. Theranostics 2018;8:1005-26.

227. Shao J, Xie H, Huang H, Li Z, Sun Z, et al. Biodegradable black phosphorus-based nanospheres for in vivo photothermal cancer therapy. Nat Commun 2016;7:12967.

228. Fu W, Zhou W, Chu PK, Yu XF. Inherent chemotherapeutic anti-cancer effects of low-dimensional nanomaterials. Chemistry (Easton) 2019;25:10995-1006. 
229. Qin L, Jiang S, He H, Ling G, Zhang P. Functional black phosphorus nanosheets for cancer therapy. J Control Release 2020;318:50-66.

230. Li Z, Zhang T, Fan F, Gao F, Ji H, et al. Piezoelectric materials as sonodynamic sensitizers to safely ablate tumors: a case study using black phosphorus. J Phys Chem Lett 2020;11:1228-38.

231. Wan S, Zhang B, Li S, He B, Pu Y. Combination of PEG-decorated black phosphorus nanosheets and immunoadjuvant for photoimmunotherapy of melanoma. J Mater Chem B 2020; doi: 10.1039/d0tb00434k.

232. Yang B, Ding L, Chen Y, Shi J. Augmenting tumor-starvation therapy by cancer cell autophagy inhibition. Adv Sci (Weinh) 2020;7:1902847.

233. Yang X, Liu G, Shi Y, Huang W, Shao J, et al. Nano-black phosphorus for combined cancer phototherapy: recent advances and prospects. Nanotechnology 2018;29:222001.

234. Aggarwal M, Saxena R, Sinclair E, Fu Y, Jacobs A, et al. Reactivation of mutant p53 by a dietary-related compound phenethyl isothiocyanate inhibits tumor growth. Cell Death Differ 2016;23:1615-27.

235. Yang H, Mao H, Wan Z, Zhu A, Guo M, et al. Micelles assembled with carbocyanine dyes for theranostic near-infrared fluorescent cancer imaging and photothermal therapy. Biomaterials 2013;34:9124-33.

236. Owens EA, Lee S, Choi J, Henary M, Choi HS. NIR fluorescent small molecules for intraoperative imaging. Wiley Interdiscip Rev Nanomed Nanobiotechnol 2015;7:828-38.

237. Xue X, Lindstrom A, Li Y. Porphyrin-based nanomedicines for cancer treatment. Bioconjug Chem 2019;30:1585-603.

238. Wang L, Chang Y, Feng Y, Li X, Cheng Y, et al. Nitric oxide stimulated programmable drug release of nanosystem for multidrug resistance cancer therapy. Nano Lett 2019;19:6800-11.

239. Charitidis CA, Georgiou P, Koklioti MA, Trompeta AF, Markakis V. Manufacturing nanomaterials: from research to industry. Manufacturing Rev 2014;1:11.

240. Khalid K, Tan X, Mohd Zaid HF, Tao Y, Lye Chew C, et al. Advanced in developmental organic and inorganic nanomaterial: a review. Bioengineered 2020;11:328-55.

241. Souto EB, Silva GF, Dias-Ferreira J, Zielinska A, Ventura F, et al. Nanopharmaceutics: part II-production scales and clinically compliant production methods. Nanomaterials (Basel) 2020;10:455.

242. Yang Y, Aw J, Xing B. Nanostructures for NIR light-controlled therapies. Nanoscale 2017;9:3698-718.

243. Zhang C, Li D, Pei P, Wang W, Chen B, et al. Rod-based urchin-like hollow microspheres of $\mathrm{Bi}_{2} \mathrm{~S}_{3}$ : Facile synthesis, photo-controlled drug release for photoacoustic imaging and chemo-photothermal therapy of tumor ablation. Biomaterials 2020;237:119835.

244. Wu S, Liu X, Ren J, Qu X. Glutathione depletion in a benign manner by $\mathrm{MoS}_{2}$-based nanoflowers for enhanced hypoxia-irrelevant freeradical-based cancer therapy. Small 2019;15:e1904870.

245. Wang R, Zhou L, Wang W, Li X, Zhang F. In vivo gastrointestinal drug-release monitoring through second near-infrared window fluorescent bioimaging with orally delivered microcarriers. Nat Commun 2017;8:14702.

246. Huang LY, Zhu S, Cui R, Zhang M. Noninvasive in vivo imaging in the second near-infrared window by inorganic nanoparticle-based fluorescent probes. Anal Chem 2020;92:535-42.

247. Tsai SR, Hamblin MR. Biological effects and medical applications of infrared radiation. J Photochem Photobiol B 2017;170:197-207.

248. Mussttaf RA, Jenkins DFL, Jha AN. Assessing the impact of low level laser therapy (LLLT) on biological systems: a review. Int J Radiat Biol 2019;95:120-43.

249. Boey A, Ho HK. All roads lead to the liver: metal nanoparticles and their implications for liver health. Small 2020; doi: 10.1002/ smll.202000153:e2000153.

250. Lindén M. Biodistribution and excretion of intravenously injected mesoporous silica nanoparticles: implications for drug delivery efficiency and safety. The Enzymes: Academic Press; 2018. pp. 155-80.

251. Wu T, Tang M. Review of the effects of manufactured nanoparticles on mammalian target organs. J Appl Toxicol 2018;38:25-40.

252. Zamboni WC, Szebeni J, Kozlov SV, Lucas AT, Piscitelli JA, et al. Animal models for analysis of immunological responses to nanomaterials: challenges and considerations. Adv Drug Deliv Rev 2018;136-137:82-96.

253. Dawidczyk CM, Russell LM, Searson PC. Recommendations for benchmarking preclinical studies of nanomedicines. Cancer Res 2015;75:4016-20.

254. Shamsi M, Mohammadi A, Manshadi MKD, Sanati-Nezhad A. Mathematical and computational modeling of nano-engineered drug delivery systems. J Control Release 2019;307:150-65.

255. Yao C, Akakuru OU, Stanciu SG, Hampp N, Jin Y, et al. Effect of elasticity on the phagocytosis of micro/nanoparticles. J Mater Chem B 2020;8:2381-92.

256. Chou TC. Drug combination studies and their synergy quantification using the Chou-Talalay method. Cancer Res 2010;70:440-6.

257. Roberti A, Valdes AF, Torrecillas R, Fraga MF, Fernandez AF. Epigenetics in cancer therapy and nanomedicine. Clin Epigenetics 2019;11:81.

258. Ai X, Hu M, Wang Z, Lyu L, Zhang W, et al. Enhanced cellular ablation by attenuating hypoxia status and reprogramming tumorassociated macrophages via NIR light-responsive upconversion nanocrystals. Bioconjug Chem 2018;29:928-38.

259. Duan M, Xia F, Li T, Shapter JG, Yang S, et al. Matrix metalloproteinase-2-targeted superparamagnetic Fe3O4-PEG-G5-MMP2@Ce6 nanoprobes for dual-mode imaging and photodynamic therapy. Nanoscale 2019;11:18426-35.

260. Moura RP, Martins C, Pinto S, Sousa F, Sarmento B. Blood-brain barrier receptors and transporters: an insight on their function and how to exploit them through nanotechnology. Expert Opin Drug Deliv 2019;16:271-85.

261. Gao W, Wang Z, Lv L, Yin D, Chen D, et al. Photodynamic therapy induced enhancement of tumor vasculature permeability using an upconversion nanoconstruct for improved intratumoral nanoparticle delivery in deep tissues. Theranostics 2016;6:1131-44. 
262. Busquets MA, Espargaró A, Sabaté R, Estelrich J. Magnetic nanoparticles cross the blood-brain barrier: when physics rises to a challenge. Nanomaterials 2015;5:2231-48.

263. Qiu Y, Tong S, Zhang L, Sakurai Y, Myers DR, et al. Magnetic forces enable controlled drug delivery by disrupting endothelial cell-cell junctions. Nat Commun 2017;8:15594.

264. Luo YH, Chang LW, Lin P. Metal-based nanoparticles and the immune system: activation, inflammation, and potential applications. Biomed Res Int 2015;2015:143720.

265. Muhammad Q, Jang Y, Kang SH, Moon J, Kim WJ, et al. Modulation of immune responses with nanoparticles and reduction of their immunotoxicity. Biomater Sci 2020;8:1490-501.

266. Baeza A. Tumor targeted nanocarriers for immunotherapy. Molecules 2020;25:1508.

267. Kobayashi H, Choyke PL. Near-infrared photoimmunotherapy of cancer. Acc Chem Res 2019;52:2332-9.

268. Luengo A, Gui DY, Vander Heiden MG. Targeting metabolism for cancer therapy. Cell Chem Biol 2017;24:1161-80.

269. Feng J, Byrne NM, Al Jamal W, Coulter JA. Exploiting current understanding of hypoxia mediated tumour progression for nanotherapeutic development. Cancers (Basel) 2019;11:E1989.

270. Bansal A, Simon MC. Glutathione metabolism in cancer progression and treatment resistance. J Cell Biol 2018;217:2291-8.

271. Benizri S, Gissot A, Martin A, Vialet B, Grinstaff MW, et al. Bioconjugated oligonucleotides: recent developments and therapeutic applications. Bioconjug Chem 2019;30:366-83.

272. Ju E, Li T, Ramos da Silva S, Gao SJ. Gold nanocluster-mediated efficient delivery of Cas9 Protein through pH-induced assemblydisassembly for inactivation of virus oncogenes. ACS Appl Mater Interfaces 2019;11:34717-24.

273. Wang P, Zhang L, Zheng W, Cong L, Guo Z, et al. Thermo-triggered release of CRISPR-Cas9 system by lipid-encapsulated gold nanoparticles for tumor therapy. Angew Chem Int Ed Engl 2018;57:1491-6.

274. Anselmo AC, Mitragotri S. Nanoparticles in the clinic: an update. Bioeng Transl Med 2019;4:e10143.

275. NIH Clinical Trials. Available from: http://clinicaltrials.gov/ct2/home. [Last accessed on 26 May 2020]

276. Rastinehad AR, Anastos H, Wajswol E, Winoker JS, Sfakianos JP, et al. Gold nanoshell-localized photothermal ablation of prostate tumors in a clinical pilot device study. Proc Natl Acad Sci U S A 2019;116:18590-6.

277. Espinosa A, Di Corato R, Kolosnjaj-Tabi J, Flaud P, Pellegrino T, et al. Duality of iron oxide nanoparticles in cancer therapy: amplification of heating efficiency by magnetic hyperthermia and photothermal bimodal treatment. ACS Nano 2016;10:2436-46. 